\title{
A New Mixed Iterative Algorithm to Solve the Fuel-Optimal Linear Impulsive
}

\section{Rendezvous Problem}

\author{
D. Arzelier · C. Louembet · A. Rondepierre · M. Kara-Zaitri
}

\begin{abstract}
The optimal fuel impulsive time-fixed rendezvous problem is reviewed. In a linear setting, it may be reformulated as a non convex polynomial optimization problem for a pre-specified fixed number of velocity increments. Relying on variational results previously published in the literature, an improved mixed iterative algorithm is defined to address the issue of optimization over the number of impulses. Revisiting the primer vector theory, it combines variational tests with sophisticated numerical tools from algebraic geometry to solve polynomial necessary and sufficient conditions of optimality. Numerical examples under circular and elliptic assumptions show that this algorithm is efficient and can be integrated into a rendezvous planning tool.
\end{abstract}

Keywords Orbital rendezvous $\cdot$ fuel optimal space trajectories $\cdot$ primer vector theory $\cdot$ impulsive maneuvers $\cdot$ linear equations of motion

\section{Introduction}

Given the increasing need for satellite servicing in current and future space programs developed in conjunction with rendezvous missions for the International Space Station (ISS), the interest of most space agencies in developing adequate rendezvous mission planning tools has been rapidly rising. In particular, new challenges have appeared relating to the synthesis of guidance schemes This work was supported by CNES Grant R-S07/VF-0001-065.

D. Arzelier, corresponding author

CNRS, LAAS, 7 avenue du colonel Roche, F-31400 Toulouse, France

D. Arzelier

Univ de Toulouse, LAAS, F-31400 Toulouse, France

E-mail: arzelier@laas.fr

C. Louembet

CNRS, LAAS, 7 avenue du colonel Roche, F-31400 Toulouse, France

C. Louembet

Univ de Toulouse, UPS, LAAS, F-31400 Toulouse, France

E-mail: clouembet@laas.fr

A. Rondepierre

IMT, 118 route de Narbonne, F-31400 Toulouse, France

A. Rondepierre

Univ de Toulouse, INSA, F-31400 Toulouse, France

E-mail: Aude.Rondepierre@insa-toulouse.fr

M. Kara-Zaïtri

CNRS, LAAS, 7 avenue du colonel Roche, F-31400 Toulouse, France

M. Kara-Zaïtri

Univ de Toulouse, LAAS, F-31400 Toulouse, France 
capable of achieving autonomous far range rendezvous on highly elliptical orbits while preserving optimality in terms of fuel consumption. Strictly speaking, the space far range rendezvous maneuver is an orbital transfer between a passive target and an actuated spacecraft called the chaser, within a fixed or floating time period. In this paper, we mainly focus on the so-called time-fixed fuel optimal rendezvous problem in a linearized gravitational field for which a renewed interest has been witnessed in the literature [1], [2], [3], [4]. Because of the constraints of on-board guidance algorithms, numerical solutions based on linear relative motion are particularly appealing. With respect to the numerical solution, direct methods based on discretizing the original problem and converting it into a linear programming problem may be used as in [2]. Indirect approaches based on the solution of optimality conditions derived from Pontryagin's Maximum Principle, leading to the development of the so-called primer vector theory presented in [5], have also been an avenue of research in numerous studies [6], [7], [8], [9]. As they only focus on fixed number and location of impulses, these approaches fail to optimize trajectory planning in terms of the number of impulsive maneuvers. Neither can they ensure the global optimality of the result provided for a fixed number of impulses. To optimize the number of impulses as well as their specific application times, an iterative algorithm based on the calculus of variations originally developed by Lion and Handelsman [10] has been designed [11,12]. The main drawback however is due to the possible non smoothness and sub-optimality of the resultant trajectory of the primer vector norm due to the local nature of the proposed approach. To overcome this difficulty, a Davidon-Fletcher-Powell penalty minimization step is proposed in order to move the impulses and achieve a smooth trajectory as detailed in [13] or [12].

The paper's contribution is to revisit the iterative algorithm of Lion-Handelsman based on the calculus of variations and to take advantage of the polynomial nature of the underlying necessary conditions to circumvent the necessity to resort to local optimization schemes. In particular, this new iterative algorithm combines the algebraic formulation of Carter's necessary and sufficient conditions, supporting the use of powerful numerical tools from the algebraic geometry field, and improving variational tests as derived in [10]. This results in a mixed different iterative strategy bypassing the local optimal search step and cusp occurrence. Circular and elliptic Keplerian reference orbits may be considered indiferrently.

In the first section of this paper, the framework of the minimum-fuel fixed-time rendezvous problem is presented and necessary and sufficient conditions of optimality are recalled. Relative dynamics motion for rendezvous are the well-known Tschauner-Hempel equations [14] and the transition matrix of Yamanaka-Ankersen [15]. The results of [10] are recalled and the mixed iterative algorithm is presented. For comparison's sake, the efficiency of the proposed algorithms is illustrated with four different numerical examples. Three academic examples taken from Carter's reference [8] are first studied in details. Also reviewed is one realistic scenario based on PRISMA which is a "technology in-orbit testbed mission" demonstrating formation flight [3]. 


\section{The Time-Fixed Optimal Rendezvous Problem}

\subsection{Linear impulsive time-fixed optimal rendezvous problem}

This paper focuses on the fixed-time minimum-fuel rendezvous between close orbits of an active (actuated) spacecraft called the chaser with a passive target spacecraft assuming a linear impulsive setting and a Keplerian relative motion as it is defined in the references [8], [9]. The impulsive approximation for the thrust means that instantaneous velocity increments are applied to the chaser whereas its position is continuous. If the relative equations of motion of the chaser are supposed to be linear and under the previous Keplerian assumptions, it is shown in the references [8], [17], [9] that the considered minimum-fuel rendezvous problem may be reformulated as the following optimization problem:

$$
\begin{array}{ll}
\min _{N, v_{i}, \Delta v_{i}, \beta\left(v_{i}\right)} J=\sum_{i=1}^{N} \Delta v_{i} \\
\text { s.t. } \\
\qquad u_{f}=\sum_{i=1}^{N} \phi^{-1}\left(v_{i}\right) B\left(v_{i}\right) \Delta v_{i} \beta\left(v_{i}\right)=\sum_{i=1}^{N} R\left(v_{i}\right) \Delta v_{i} \beta\left(v_{i}\right) \\
& \left\|\beta\left(v_{i}\right)\right\|=1 \\
& \Delta v_{i} \geq 0
\end{array}
$$

where $\phi(v)$ is the fundamental matrix associated to the linearized relative free motion and $\Phi\left(v, v_{1}\right)=\phi(v) \phi^{-1}\left(v_{1}\right)$ denotes therefore the transition matrix of the linearized relative free motion. Note that the true anomaly $v$ has been chosen as the independent variable throughout in the paper. $v_{0}$ and $v_{f}$ denote respectively the initial and final values of the true anomaly during the rendezvous. $u_{f}=\phi^{-1}\left(v_{f}\right) X_{f}-\phi^{-1}\left(v_{1}\right) X_{0} \neq 0$ where the state vector $X_{f}=\left[r_{f}^{T} v_{f}^{T}\right]^{T}$ at $v_{f}$ and the state vector $X_{0}=\left[\begin{array}{ll}r_{0}^{T} & v_{0}^{T}\end{array}\right]^{T}$ at $v_{0}$ are composed of the relative positions and relative velocities vectors. The optimization decision variables are the number of impulses $N$, the sequence of thrust times $\left\{v_{i}\right\}_{i=1, \cdots, N}$, the sequence of thrust magnitudes $\left\{\Delta v_{i}\right\}_{i=1, \cdots, N}$ and of thrust directions $\left\{\beta\left(v_{i}\right)\right\}_{i=1, \cdots, N}$. Due to the lack of a priori information about the optimal number of impulses to be considered, problem (1) is very hard to solve from both theoretical and numerical points of view. Therefore, the associated fixed-time minimum-fuel rendezvous problem for a fixed number $N$ of impulses has been considered in the literature mainly via geometric methods near circular [6], [13], [18] or elliptic [17] orbits. These results are mainly based on the derivation of optimality conditions for the problem (1) when $N$ is fixed a priori.

\subsection{Carter's necessary and sufficient conditions for a fixed number of impulses}

When the number of impulses is not a part of the optimization process and is fixed a priori to $N$, problem (1) may be considered as the joint optimal selection of $N$ velocity increments $\Delta V\left(v_{i}\right)=\Delta v_{i} \beta\left(v_{i}\right)$ and $N$ times $v_{i}$ of maneuvers. Applying a Lagrange multiplier rule for the problem (1) as in [17], one can derive necessary conditions of optimality (2) to (6) in terms of the Lagrange multiplier vector $\lambda \in \mathbb{R}^{n}$ as is recalled in theorem 2.1 below. Prussing has first shown in [19] that these conditions are 
also sufficient in the case of linear relative motion with the strengthening semi-infinite constraint (9) that should be fulfilled on the continuum $\left[v_{0}, v_{f}\right]$ and is expressed in terms of the transition matrix $R(v)$.

\section{Theorem 2.1 [9]}

$\left(v_{1}, \ldots, v_{N}, \Delta v_{1}, \ldots, \Delta v_{N}, \beta\left(v_{1}\right), \ldots, \beta\left(v_{N}\right)\right)$ is the optimal solution of problem (1) if and only if there exists a non-zero vector $\lambda \in \mathbb{R}^{m}, m=\operatorname{dim}(\phi)$ that verifies the necessary and sufficient conditions:

$$
\begin{gathered}
\Delta v_{i}=0 \text { or } \beta\left(v_{i}\right)=R^{T}\left(v_{i}\right) \lambda, \forall i=1, \cdots, N \\
\Delta v_{i}=0 \text { or } \lambda^{T} R\left(v_{i}\right) R\left(v_{i}\right)^{T} \lambda=1, \forall i=1, \cdots, N \\
\Delta v_{i}=0 \text { or } v_{1}=v_{0} \text { or } v_{N}=v_{f} \text { or } \lambda^{T} \frac{d R\left(v_{i}\right)}{d v} R\left(v_{i}\right)^{T} \lambda=0, \forall i=1, \cdots, N \\
\sum_{i=1}^{N}\left[R\left(v_{i}\right) R^{T}\left(v_{i}\right)\right] \lambda \Delta v_{i}=u_{f} \\
\Delta v_{i} \geq 0, \forall i=1, \cdots, N \\
\sum_{i=1}^{N} \Delta v_{i}=u_{f}^{T} \lambda>0
\end{gathered}
$$

$u_{f}^{T} \lambda$ is the minimum of the set defined as : $\left\{\lambda \in \mathbb{R}^{m}:(2)-(7)\right.$ are verified $\}$

$$
\left\|\lambda_{v}(v)\right\| \leq 1 \quad \forall v \in\left[v_{1}, v_{N}\right]
$$

where $\lambda_{v}(v)=R(v)^{T} \lambda$ denotes the so-called primer vector. Note that conditions (7) and (8) may be easily derived from the previous ones. These results derive directly from the seminal work of [5] in the early sixties and form an alternative formulation to the primer vector theory. The primer vector is nothing but the co-state vector associated with the relative velocity and is related to the Lagrange multiplier vector through the preceding relation.

A numerical solution of optimality conditions (2) to (9) in the unknowns $\lambda \in \mathbb{R}^{m},\left\{v_{i}\right\}_{i=1, \cdots, N},\left\{\beta\left(v_{i}\right)\right\}_{i=1, \cdots, N},\left\{\Delta v_{i}\right\}_{i=1, \cdots, N}$ is still hard to find for a fixed number of impulses $N$ due to the non convex and transcendental nature of these polynomial equalities and inequalities. However, it is shown in [20] that the problem may be tackled by using an adequate dynamic gridding strategy and polynomial optimization. Still, the optimal number of impulses $N^{*}$ for a particular rendezvous problem is in general unknown and only a bound $N^{*} \leq N_{\max }$ is available [21]. $N_{\max }=2$ for out-of-plane rendezvous, $N_{\max }=4$ for in-plane rendezvous while $N_{\max }=6$ for a general three-dimensional rendezvous problem. Apart the gridding step, the necessity to try every case for $2 \leq N \leq N_{\max }$ in [20] appears be very time-consuming and a more direct approach to solve problem (1) is now proposed. 


\section{Optimizing over the Number of Impulses}

3.1 Using Lion \& Handelsman results on multi-impulse trajectories

In [10], a method is proposed to take advantage of the primer vector theory developed by Lawden in order to improve non optimal trajectories by adding or shifting impulses. The calculus of variations is used to find conditions on the norm of the primer vector for an additional impulse and on the derivative of this norm for initial and/or final coastings. The method is mainly based on derivation of the so-called variational adjoint equation resulting from the variation of the cost function. Later, Jezewski [11], [12] developed a numerical algorithm combining Lion-Handelsman's conditions with a modified gradient search approach in a linear model setting. The additional local optimization procedure is used to find the optimal position and modulus of the additional impulse so as to avoid a resulting cusp for the norm of the primer vector as reported in [11], [12]. In this section, the conditions of Lion and Handelsman are recalled and a different Heuristic iterative procedure avoiding local optimal search step and cusp occurrence is proposed. It is worth noticing that the extension of these conditions for elliptical reference orbit (Tschauner-Hempel [14] dynamical relative model and Yamanaka-Ankersen transition matrix [15]) from [16] is used here.

\subsubsection{Additional interior impulse condition}

Perturbing a reference initial two-impulse trajectory and adding an interior impulse at $v_{m}$, the differential cost can be expressed as:

$$
\delta J=\Delta v_{m}\left(1-\lambda_{v}\left(v_{m}\right)^{T} \beta\left(v_{m}\right)\right)=\Delta v_{m}\left(1-\lambda^{T} R\left(v_{m}\right) \beta\left(v_{m}\right)\right)
$$

From (10), it is easy to conclude that $\delta J<0$ when $\lambda_{v}\left(v_{m}\right)>1$ and that a maximum decrease in cost is obtained when:

$$
v_{m}=\arg \max _{v \in\left[v_{0}, v_{f}\right]}\left\|\lambda_{v}(v)\right\|=\arg \max _{v \in\left[v_{0}, v_{f}\right]}\left\|R^{T}(v) \lambda\right\|
$$

\subsubsection{Additional coasting period conditions}

For an additional initial coasting period of duration $d v_{1}$, the cost variation is given by:

$$
\delta J=-\Delta v_{1} \frac{d \lambda_{v}{ }^{T}}{d v}\left(v_{1}\right) \lambda_{v}\left(v_{1}\right) d v_{1}=-\Delta v_{1} \lambda^{T} \frac{d R}{d v}\left(v_{1}\right) R^{T}\left(v_{1}\right) \lambda d v_{1}=\Delta v_{1}^{2} \frac{d\left\|\lambda_{v}(v)\right\|}{d v} d v=v_{1} d v_{1}
$$

This condition means that adding an initial coasting arc of $d v_{1}>0$ duration may improve the cost if $\dot{\lambda}_{v}\left(v_{1}\right)^{T} \lambda_{v}\left(v_{1}\right)>0$, i.e., the right derivative of the primer vector norm at $v_{1}$ is positive. Similarly, for a final coasting arc of duration $d v_{f}$, we get:

$$
\delta J=-\Delta v_{f} \frac{d \lambda_{v}}{d v}\left(v_{f}\right)^{T} \lambda_{v}\left(v_{f}\right) d v_{f}=-\Delta v_{f} \lambda^{T} \frac{d R}{d v}\left(v_{f}\right) R^{T}\left(v_{f}\right) \lambda d v_{f}=-\Delta v_{f}^{2} \frac{d\left\|\lambda_{v}(v)\right\|}{d v} \underset{\mid v=v_{f}}{ } d v_{f}
$$


A final coast of $d v_{f}<0$ duration will improve the cost when the left derivative of the primer vector norm at $v_{f}$ is negative, i.e., when $\frac{d \lambda_{v}}{d v}\left(v_{f}\right)^{T} \lambda_{v}\left(v_{f}\right)$

\subsection{A mixed iterative algorithm}

These conditions may be used jointly to reduce the cost of a reference non optimal two-impulse trajectory but can also be generalized to multi-impulse trajectories. Consider the four-impulse trajectory of Fig. 1a. Overall cost can be reduced by considering the coasting arc $\left[v_{i}, v_{i+1}\right]$ in different ways:

- Adding a new impulse at $v_{m}$,

- Adding an initial coasting arc by shifting $v_{i}$ toward $v_{m}$, if $\frac{d\|\lambda\|}{d v}\left(v_{i}\right)>0$,

- Adding a final coasting arc by shifting $v_{i+1}$ toward $v_{m}$, if $\frac{d\|\lambda\|}{d v}\left(v_{i+1}\right)<0$,

- Replacing $v_{i}$ and $v_{i+1}$ by $v_{m}$, if $\frac{d\|\lambda\|}{d v}\left(v_{i}\right)>0$ and $\frac{d\|\lambda\|}{d v}\left(v_{i+1}\right)<0$. This is equivalent to adding an initial and a final coasting $\operatorname{arc}$ on $\left[v_{i}, v_{i+1}\right]$ and an impulse at $v_{m}$.

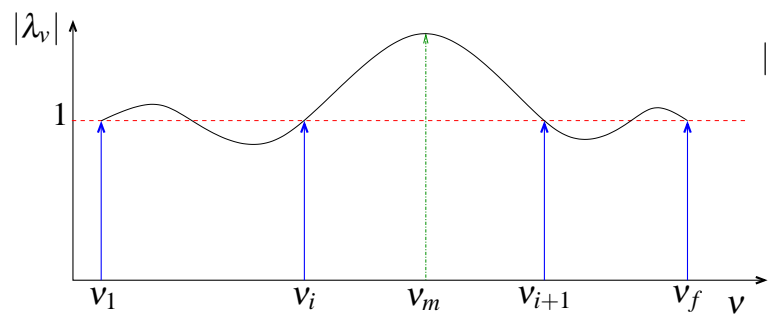

(a) Adding or moving impulses on a multi-impulse trajectory

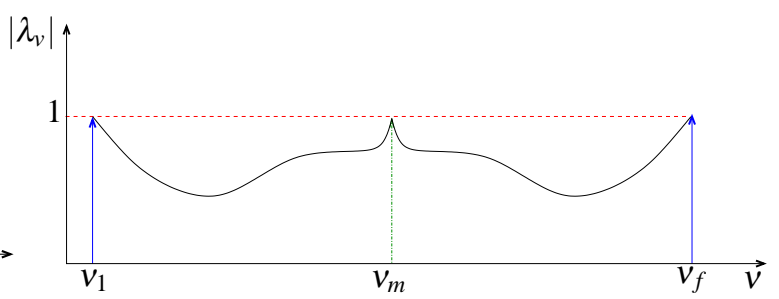

(b) Nonoptimal primer vector norm with a cusp at the interior impulse

Fig. 1: Nonoptimal primer vector norms

As noted in [11] and in [22], computation of the mid-impulse might nevertheless result in a non optimal trajectory not verifying the optimality conditions of Lawden and condition (9), particularly in the case of occurrence of a cusp at $v_{m}$ as illustrated in Fig. 1b. A particular strategy combining Lion-Handelsman's conditions and local direct optimization based on the Davidon-Fletcher-Powell penalty method in [12] or based on BFGS method in [22] has been proposed to optimize the resulting three-impulse trajectory. The objective of this section is to propose an alternative to this complicated procedure by developing a mixed iterative algorithm taking advantage of the algebraic formulation of Carter's optimality conditions and of the LionHandelsman's conditions. Starting from a non optimal two-impulse trajectory, successive admissible improved trajectories will be iteratively built by:

- Adding impulse at $v_{m}$ if the impulse number does not exceed the upper bound $N_{\max }$;

- Moving the proximal impulse to $v_{m}$ if an impulse cannot be added due to $N=N_{\text {max }}$;

- Merging two impulses at $v_{m}$ if there is no proximal impulse and if an impulse cannot be added due to $N=N_{\max }$. 
where $v_{m}$ is defined by (11). The logic of the proposed heuristic algorithm is depicted at Figure 2 where $d p_{i}=\frac{d\|\lambda\|}{d v}\left(v_{i}\right)$ and $d p_{i+1}=\frac{d\|\lambda\|}{d v}\left(v_{i+1}\right)$. The set $T_{i m p}$ denotes the current set impulses and the new impulse $v_{m}$ is always added to it.

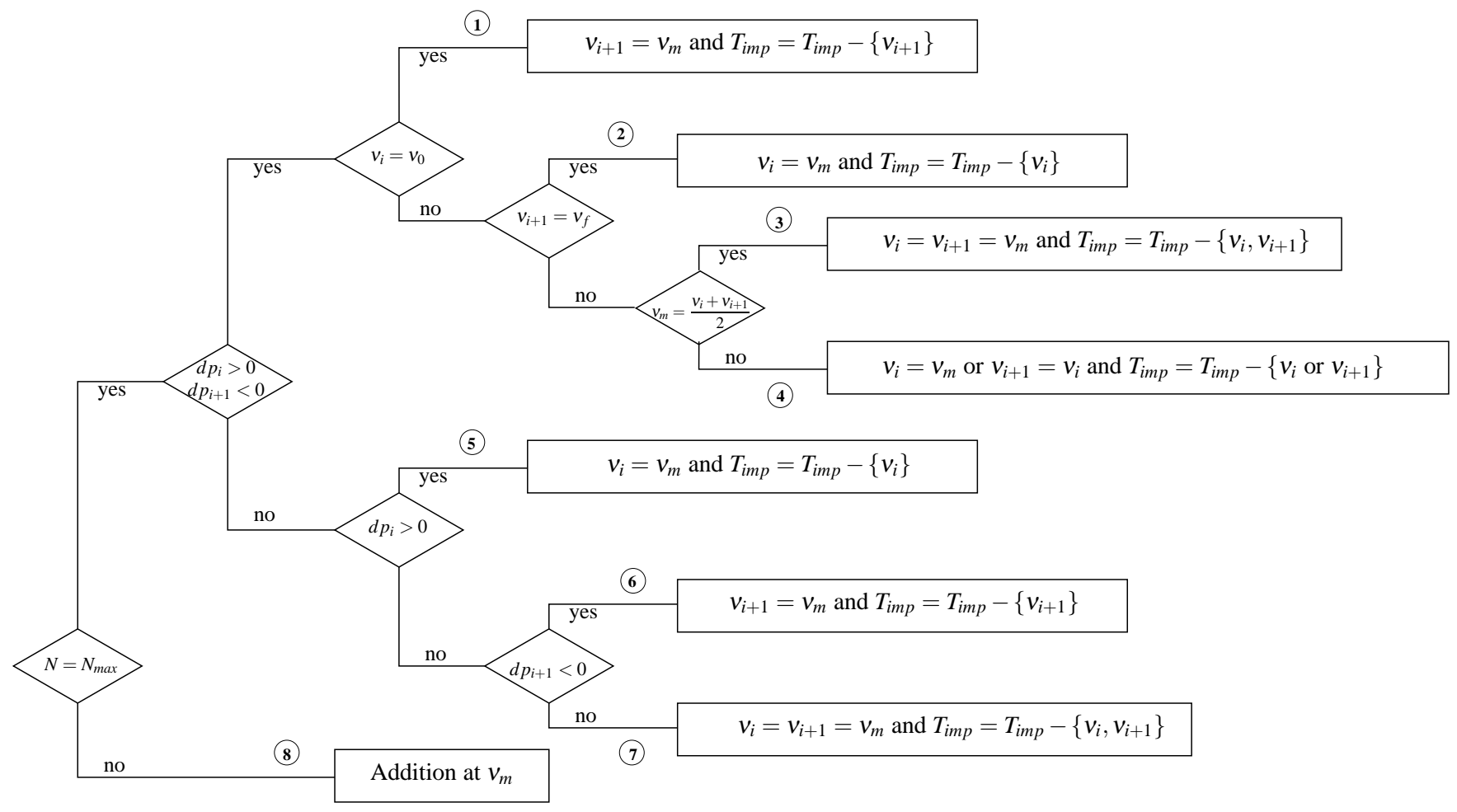

Fig. 2: Heuristic for the iterative mixed algorithm

This heuristic procedure relies on basic principles that are used to make the successive sequences of maneuvers monotonically converging to an optimal solution.

- The first and final maneuvers time defined respectively at $v_{0}$ and $v_{f}$ cannot be moved in the process. Therefore, optimal solutions consisting of an initial and/or final coasting period cannot be found by this algorithm.

- A new impulse is always added at $v_{m}$. In case 8 for an actual number of impulses $N<N_{\max }$, it increases the number of impulses while when $N=N_{\max }$ it reduces the number of impulses in cases 3 and 7 and it does not change this number for cases $1,2,4,5$ and 6.

- Every move of an actual maneuver time to $v_{m}$ is chosen to be the proximal of $v_{m}$ (in particular for case 4) except in case 7 which is specific since moves of impulses $v_{i}$ and $v_{i+1}$ are not required to improve the trajectory. In this case, the idea is rather to re-initialize the iterative process with a new three-impulse trajectory. In practice, this case has never occurred on all the tested numerical examples.

The systematic convergence of the algorithm for any rendezvous is not analytically proved but no such case has been reported in the different numerical tests performed so far.

The algorithm may now be described in details. It is mainly composed of two stages: one initialization step solving a twoimpulse rendezvous problem and the iterative procedure building the final plan of maneuvers. $\varepsilon_{\text {cond }}$ and $\varepsilon_{\lambda}$ are respectively 
precision values on the conditioning number of the transition sub-matrix $\Phi_{12}$ and on the maximum of the norm of the primer vector. Typically, $\varepsilon_{\text {cond }}=10^{6}$ while $\varepsilon_{\lambda}=10^{-6}$.

\section{Initialization step:}

a. Solve the two-impulse problem:

\section{1- Initialize}

$$
T_{\text {imp }}=\left\{v_{1}, v_{2}\right\}=\left\{v_{0}, v_{f}\right\}
$$

2- Compute the transition matrices

$$
\Phi\left(v_{f}, v_{1}\right)=\left[\begin{array}{c}
\Phi_{11}\left(v_{f}, v_{1}\right) \Phi_{12}\left(v_{f}, v_{1}\right) \\
\Phi_{21}\left(v_{f}, v_{1}\right) \Phi_{22}\left(v_{f}, v_{1}\right)
\end{array}\right], \Phi^{\#}\left(v_{f}, v_{1}\right)=\Phi^{-T}\left(v_{f}, v_{1}\right)=\left[\begin{array}{c}
\Phi_{11}^{\#}\left(v_{f}, v_{1}\right) \Phi_{12}^{\#}\left(v_{f}, v_{1}\right) \\
\Phi_{21}^{\#}\left(v_{f}, v_{1}\right) \Phi_{22}^{\#}\left(v_{f}, v_{1}\right)
\end{array}\right]
$$

If cond $\left(\Phi_{12}\left(v_{f}, v_{1}\right)\right)<\varepsilon_{\text {cond }} \underline{\text { Then }}$

$$
\begin{aligned}
& \Delta V\left(v_{1}\right)=\Phi_{12}^{-1}\left(v_{f}, v_{1}\right)\left[r_{f}-\Phi_{11}\left(v_{f}, v_{1}\right) r_{1}-\Phi_{12}\left(v_{f}, v_{1}\right) v_{1}\right] \\
& \Delta V\left(v_{f}\right)=v_{f}+\left[\Phi_{22}\left(v_{f}, v_{1}\right) \Phi_{12}^{-1}\left(v_{f}, v_{1}\right) \Phi_{11}\left(v_{f}, v_{1}\right)-\Phi_{21}\left(v_{f}, v_{1}\right)\right] r_{1}-\Phi_{22}\left(v_{f}, v_{1}\right) \Phi_{12}^{-1}\left(v_{f}, v_{1}\right) r_{f}
\end{aligned}
$$

$\underline{\text { Else }}$

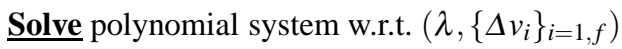

$$
\begin{aligned}
\lambda^{T} R\left(v_{i}\right) R\left(v_{i}\right)^{T} \lambda & =1, \forall v_{i} \in T_{i m p} \\
\sum_{v_{i} \in T_{\text {imp }}}\left[R\left(v_{i}\right) R^{T}\left(v_{i}\right)\right] \lambda \Delta v_{i} & =u_{f}, \forall v_{i} \in T_{i m p}, i=1, f \\
\Delta v_{i} & \geq 0, i=1, f
\end{aligned}
$$

Choose the minimum-fuel solution:

$$
\lambda_{\text {init }}=\arg \left[\min _{\lambda} u_{f}^{T} \lambda\right]
$$

Compute impulses:

$$
\begin{gathered}
\beta\left(v_{i}\right)=R\left(v_{i}\right)^{T} \lambda_{\text {init }}, \forall v_{i} \in T_{\text {imp }}, i=1, f \\
\Delta V\left(v_{i}\right)=\Delta v_{i} \beta\left(v_{i}\right), \forall v_{i} \in T_{\text {imp }}, i=1, f
\end{gathered}
$$

b. Propagate primer vector $\lambda_{v}(v)$ on a grid $\Pi=\left\{v_{1}, \cdots v_{f}\right\}$ :

If cond $\left(\Phi_{21}^{\#}\left(v_{f}, v_{1}\right)\right)<\varepsilon_{\text {cond }} \underline{\text { Then }}$

$$
\begin{aligned}
& \lambda_{v}\left(v_{1}\right)=\frac{\Delta V\left(v_{1}\right)}{\Delta v_{1}} \quad \lambda_{v}\left(v_{f}\right)=\frac{\Delta V\left(v_{f}\right)}{\Delta v_{f}} \\
& \lambda_{v}(v)=\Phi_{21}^{\#}\left(v, v_{1}\right) \Phi_{21}^{\#-1}\left(v_{f}, v_{1}\right)\left[\lambda_{v}\left(v_{f}\right)-\Phi_{22}^{\#}\left(v_{f}, v_{1}\right) \lambda_{v}\left(v_{1}\right)\right]+\Phi_{22}^{\#}\left(v, v_{1}\right) \lambda_{v}\left(v_{1}\right)
\end{aligned}
$$


$\underline{\text { Else }}$

Compute $R(v)$ on grid $\Pi$ and Propagate $\lambda_{v}(v)$ via

$$
\lambda_{v}(v)=R^{T}(v) \lambda
$$

\section{c. Compute}

$$
\lambda_{v m}=\max _{v \in\left[v_{0}, v_{f}\right]}\left\|\lambda_{v}(v)\right\| \text { and } v_{m}=\arg \left[\max _{v \in\left[v_{0}, v_{f}\right]}\left\|\lambda_{v}(v)\right\|\right]
$$

d. If $\lambda_{v m}-1<\varepsilon_{\lambda}$ Then stop. The two-impulse trajectory is optimal.

$\underline{\text { Else }}$ start iterative procedure.

$\varepsilon_{1 \lambda}$ and $\varepsilon_{2 \lambda}$ are two different precision values used in the iterative procedure to test the maximum value of the norm of the primer vector with respect to $1 . \varepsilon_{\text {cost }}$ is a parameter used to check the evolution of the cost during the iterative process.

\section{Iterative procedure:}

$\underline{\text { While }}\left(\lambda_{v m}-1 \geq \varepsilon_{1 \lambda}\right)$ and $\left(\operatorname{diff}_{\text {cost }}=\left|u_{f}^{T} \lambda_{\text {iter }}-u_{f}^{T} \lambda_{\text {iter-1 }}\right|>\varepsilon_{\text {cost }}\right.$ or $\left.\lambda_{v m}-1 \geq \varepsilon_{2 \lambda}\right)$

a. iter $\leftarrow$ iter $+1 ; \underline{\text { Choose }} v_{a}, v_{b} \in T_{i m p}$ such that

$$
\left(v_{a}<v_{m}<v_{b}\right) \text { and }\left(v_{a}=v_{i}, v_{b}=v_{i+1}\right)
$$

b. Modify $T_{i m p}=T_{i m p} \cup\left\{v_{m}\right\}$.

If $\operatorname{dim}\left(T_{i m p}\right)>N_{\max } \underline{\text { Then }} \underline{\text { Modify }} T_{i m p}$ as

(1) $\underline{\text { If }} \frac{d \lambda_{v}\left(v_{a}\right)^{T}}{d v} \lambda_{v}\left(v_{a}\right)>0$ and $\frac{d \lambda_{v}\left(v_{b}\right)^{T}}{d v} \lambda_{v}\left(v_{b}\right)<0 \underline{\text { Then }}$

(i) If $v_{a}=v_{1} \underline{\text { Then }}$

$$
v_{b}=v_{m} \text { and } T_{i m p}=T_{i m p}-\left\{v_{b}\right\}
$$

(ii) $\underline{\text { Else }}$

$$
\text { If } v_{b}=v_{f} \underline{\text { Then }}
$$

$$
v_{a}=v_{m} \text { and } T_{i m p}=T_{i m p}-\left\{v_{a}\right\}
$$


(iii) Else

$$
v_{a}=v_{b}=v_{m} \text { and } T_{i m p}=T_{i m p}-\left\{v_{a}, v_{b}\right\} \text { if } v_{m}=\left(v_{a}+v_{b}\right) / 2
$$

$$
v_{a} \text { or } v_{b}=v_{m} \text { and } T_{i m p}=T_{i m p}-\left\{v_{a} \text { or } v_{b}\right\} \text { if }\left|v_{m}-v_{a}\right|<\left|v_{m}-v_{b}\right| \text { or }\left|v_{m}-v_{b}\right|<\left|v_{m}-v_{a}\right|
$$

(2) $\underline{\text { If }} \frac{d \lambda_{v}\left(v_{a}\right)^{T}}{d v} \lambda_{v}\left(v_{a}\right)<0 \underline{\text { or }} \frac{d \lambda_{v}\left(v_{b}\right)^{T}}{d v} \lambda_{v}\left(v_{b}\right)>0$

(i) $\underline{\text { If }} \frac{d \lambda_{v}\left(v_{a}\right)^{T}}{d v} \lambda_{v}\left(v_{a}\right)>0 \underline{\text { Then }}$

$$
v_{a}=v_{m} \text { and } T_{i m p}=T_{i m p}-\left\{v_{a}\right\}
$$

(ii) $\underline{\text { If }} \frac{d \lambda_{v}\left(v_{b}\right)^{T}}{d v} \lambda_{v}\left(v_{b}\right)<0 \underline{\text { Then }}$

$$
v_{b}=v_{m} \text { and } T_{i m p}=T_{i m p}-\left\{v_{b}\right\}
$$

(iii) $\underline{\text { If }}{\frac{d \lambda_{v}\left(v_{b}\right)^{T}}{d v}}^{T} \lambda_{v}\left(v_{b}\right)>0 \underline{\text { and }}{\frac{d \lambda_{v}\left(v_{a}\right)^{T}}{d v}}^{T} \lambda_{v}\left(v_{a}\right)<0$

$$
v_{a}=v_{b}=v_{m} \text { and } T_{i m p}=T_{i m p}-\left\{v_{a}, v_{b}\right\}
$$

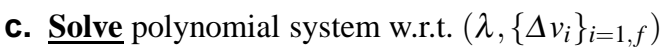

$$
\begin{aligned}
\lambda^{T} R\left(v_{i}\right) R\left(v_{i}\right)^{T} \lambda & =1, \forall v_{i} \in T_{\text {imp }} \\
\sum_{v_{i} \in T_{i m p}}\left[R\left(v_{i}\right) R^{T}\left(v_{i}\right)\right] \lambda \Delta v_{i} & =u_{f}, \forall v_{i} \in T_{i m p}, i=1, \cdots, N \\
\Delta v_{i} & \geq 0, i=1, \cdots, N
\end{aligned}
$$

d. Choose the minimum-fuel solution:

$$
\lambda_{\text {iter }}=\arg \left[\min _{\lambda} u_{f}^{T} \lambda\right]
$$

e. Compute impulses:

$$
\begin{gathered}
\beta\left(v_{i}\right)=R\left(v_{i}\right)^{T} \lambda_{\text {iter }}, \forall v_{i} \in T_{\text {imp }}, i=1, \cdots, N \\
\Delta V\left(v_{i}\right)=\Delta v_{i} \beta\left(v_{i}\right), \forall v_{i} \in T_{\text {imp }}, i=1, \cdots, N
\end{gathered}
$$

f. Compute cost difference

$$
\operatorname{diff}_{\mathrm{cost}}=\left|u_{f}^{T} \lambda_{\text {iter }}-u_{f}^{T} \lambda_{\text {iter }-1}\right|
$$

g. Compute $R(v)$ on grid $\Pi$ and Propagate $\lambda_{v}(v)$ via

$$
\lambda_{v}(v)=R^{T}(v) \lambda_{\text {iter }}
$$




\section{h. Compute}

$$
\lambda_{v m}=\max _{v \in\left[v_{1}, v_{f}\right]}\left\|\lambda_{v}(v)\right\| \text { and } v_{m}=\arg \left[\max _{v \in\left[v_{1}, v_{f}\right]}\left\|\lambda_{v}(v)\right\|\right]
$$

\section{Repeat Iterative Procedure until $\lambda_{v m} \leq 1$}

Initialization stage and step $c$ of iterative procedure require solving a system of polynomial equations ((17) in the first case if the transition matrix $\Phi\left(v_{f}, v_{1}\right)$ is ill-conditioned and (29) in the iterative procedure) with respect to $\lambda$ and $\Delta v_{i}$. The set of solutions to the two first set of equations of this polynomial system is composed of 8 couples of solutions $\left(\lambda,\left\{\Delta v_{i}\right\}_{i=1, \cdots, f}\right)$. Among this set of solutions, only those corresponding to a positive magnitude are kept to compute the minimum-fuel solution. Note that regular algebraic tools for finding all real solutions of multivariate polynomial equations based on formal Gröbner basis computation may fail due to highly complex equations. Here, homotopy continuation methods have been used [23]. In particular, the free software package PHCPack developed by Jan Verschelde [24] is used to solve the system of polynomial equations at each iteration at step $c$ of the iterative procedure and at the initialization step if necessary. The efficiency of this algorithm is now demonstrated on several different examples.

\section{Applications and Numerical Examples}

In this section, numerical results obtained from the mixed iterative algorithm are compared with previous ones published in the literature [8] and on a more realistic case borrowed from the PRISMA test bed [3]. When the mixed iterative algorithm converges to an $N_{i t}$ solution, then the PRDV algorithm from [20] is used to certify optimality of this solution for this fixed number of impulses. Only coplanar elliptic rendezvous problems based on the Yamanaka-Ankersen transition matrix [15] are considered for numerical illustration of the results proposed. Note also that the algorithm has been successfully applied to the highly elliptic rendezvous mission SIMBOL-X in [16]. In this case and under Keplerian assumptions, the bound of Neustadt [21] on the optimal number of impulses is 4 and therefore $N_{\max }=4$ in the following. Note that for the first three particular cases in which eccentricity $e=0$, the Yamanaka-Ankersen transition matrix reduces to the Hill-Clohessy-Wiltshire transition matrix [25]. Finally, all numerical examples are processed using PHCpack 2.3.52 [23], [24] under Matlab 2010b(c) running on an Intel@ Core(TM) i7 X920 2.0GHz system with 8GB ram.

\subsection{Case study 1}

The first numerical example is defined by the third case first considered in the reference [8] and later in [20] via polynomial optimization. Data given in Table 1 define a circle-to-circle rendezvous problem where the chaser is one unit before the target with the same velocity and has to rendezvous with the target with a positive vertical velocity after one orbital period. 


\begin{tabular}{|c|c|}
\hline Eccentricity & $e=0$ \\
\hline$v_{0}$ & $0 \mathrm{rad}$ \\
\hline$X_{0}^{T}$ & $\left.\begin{array}{llll}1 & 0 & 0 & 0\end{array}\right]$ \\
\hline$v_{f}$ & $2 \pi \mathrm{rad}$ \\
\hline$X_{f}^{T}$ & {$\left[\begin{array}{lllll}0 & 0 & 0 & 0.427\end{array}\right.$} \\
\hline
\end{tabular}

Table 1: Data for Carter's third example

The mixed iterative algorithm reaches the optimal four-impulse solution within 16 seconds after 6 iterations with an initial and a final impulse. For comparison's sake, the Table 2 recalls the results given in the references [8] and [20] alongside the ones given by the mixed iterative algorithm and obtained from the application of the procedure given in [18]. Remember that Carter's results are obtained using a priori pre-assigned thrust times. The results of the mixed iterative algorithm may be certified via the comparison with those of [20] and by applying the quadratic-based method proposed in [18] to build 4-impulse optimal solutions for circular rendezvous.

\begin{tabular}{|c|c|c|c|c|}
\hline & Carter [8] & Mixed iterative algorithm & PRDV algorithm [20] & Analytic procedure [18] \\
\hline$v_{\text {int }}^{1}(\mathrm{rad})$ & $\frac{\pi}{2} \simeq 1.5708$ & 1.7016 & 1.7077 & 1.70033 \\
\hline$v_{\text {int }}^{2}(\mathrm{rad})$ & $\frac{3 \pi}{2} \simeq 4.7123$ & 4.58137 & 4.5859 & 4.58286 \\
\hline$\Delta V\left(v_{0}\right)^{T}$ & {$[-0.027290 .03436]$} & {$[-0.026260 .03281]$} & {$[-0.026550 .0329]$} & {$[-0.026270 .03282]$} \\
\hline$\Delta V\left(v_{1}\right)^{T}$ & {$[0.089650 .01194]$} & {$[0.09140 .00439]$} & {$[0.09170 .004614]$} & {$[0.091390 .00447]$} \\
\hline$\Delta V\left(v_{2}\right)^{T}$ & {$[0.39010 .01194]$} & {$[-0.09140 .00439]$} & {$[-0.090110 .00434]$} & {$[-0.091390 .004472]$} \\
\hline$\Delta V\left(v_{f}\right)^{T}$ & {$[0.027290 .03436]$} & {$[0.0226260 .03281]$} & {$[0.02590 .0322]$} & {$[0.023270 .03282]$} \\
\hline $\mathscr{L}_{1} / l_{2}$ cost & 0.2686 & 0.267085 & 0.2667 & 0.267085 \\
\hline
\end{tabular}

Table 2: Results comparison for the third case of [8]

Note that the results of PRDV algorithm are rather optimistic in terms of consumption due to numerical approximations in the computation process leading to a solution that does not respect exactly the symmetry requirements of four-impulse optimal solutions [18]. Norm of the primer vector, thrust directions, primer vector locus, cost evolution during the iterative process are depicted on Figure 3. 

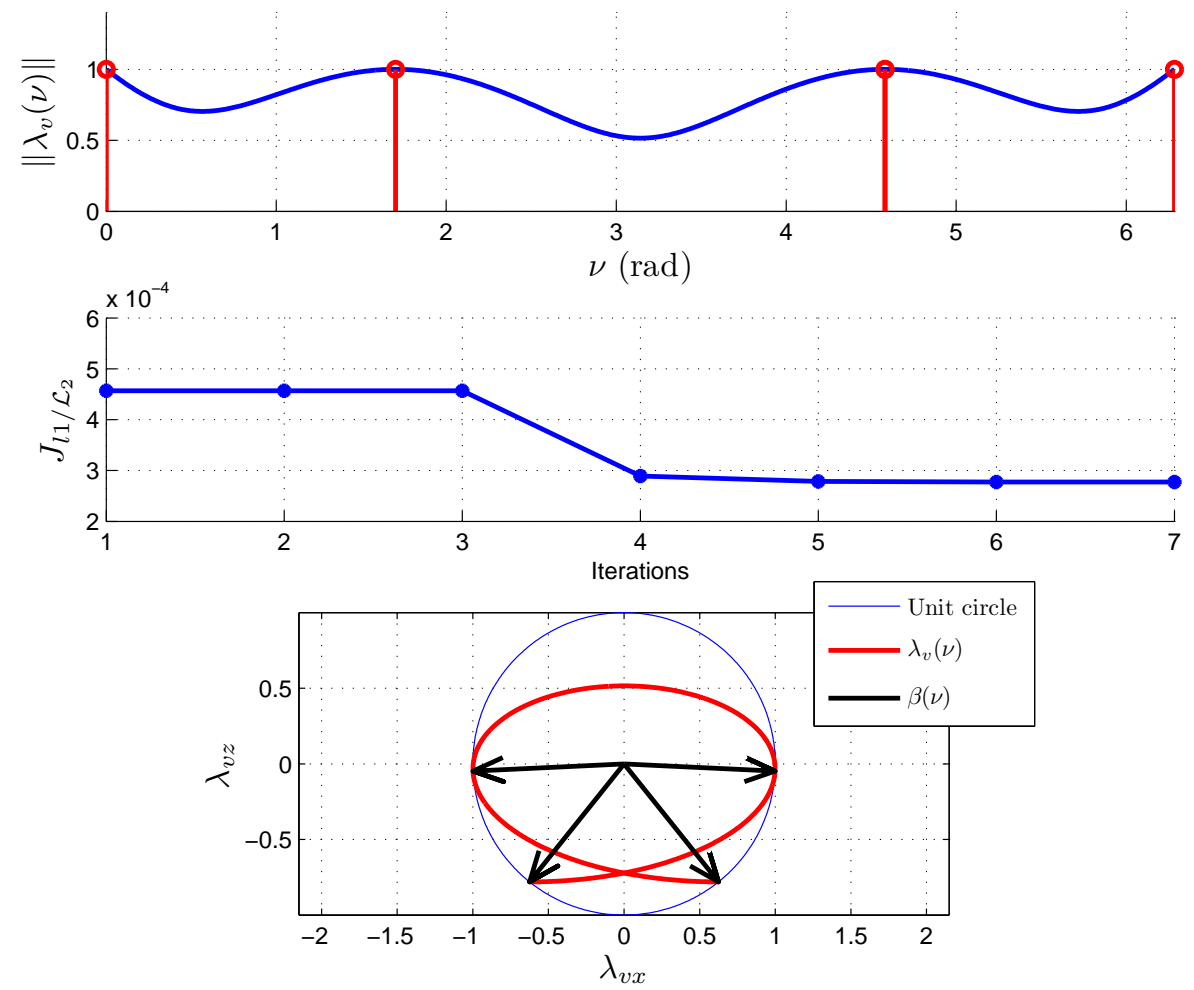

Fig. 3: $\left\|\lambda_{v}(v)\right\|$, primer vector locus, $\mathscr{L}_{1} / l_{2}$ cost for the third case of Carter's example [8]

Figures 4 to 5 show the iterative construction of the optimal solution following the proposed strategy. Note that the norm of the primer vector $\left\|\lambda_{v}(v)\right\|$ is a smooth function of the anomaly at every step of the process, thereby overcoming the main drawback of the usual iterative procedure originally proposed by Lion-Handelsman [10] and developed lately in [12] and [11]. Red impulses give the localization of the thrusts effectively computed at the preceding step while the green impulse gives the localization of the resulting impulse from either addition of a new impulse or move of a red impulse.

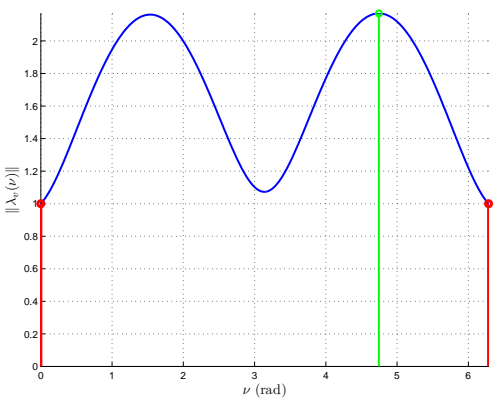

(a) Iteration 1: $v_{m}=4.7438$

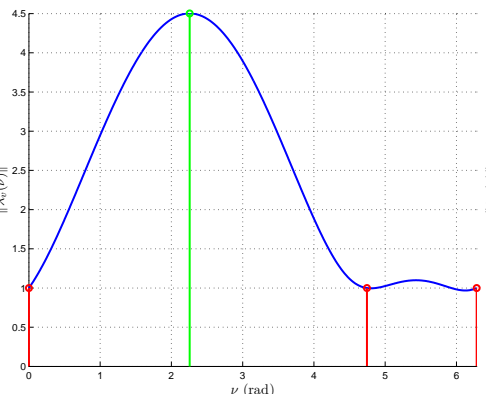

(b) Iteration 2: $v_{m}=2.25671$

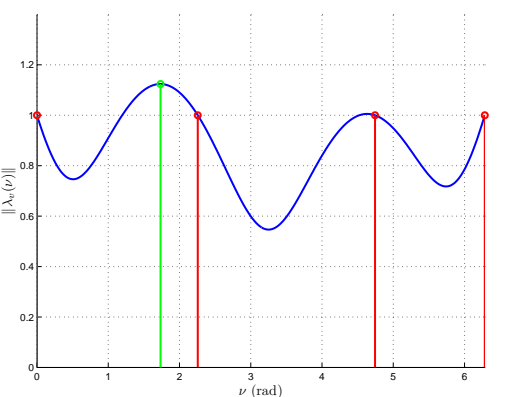

(c) Iteration 3: $v_{b}=v_{m}=1.7331$

Fig. 4: Details of iterations 1-3 for the third case of Carter [8]

The whole process is formed of two separate stages as by is easily deduced from the analysis of these details. The first stage consists mainly in a successive addition of new impulses from the initial two-impulse solution to a non-optimal four-impulse solution. Once the upper-bound of Neustadt is reached, the algorithm moves the interior impulses to find their optimal location. 


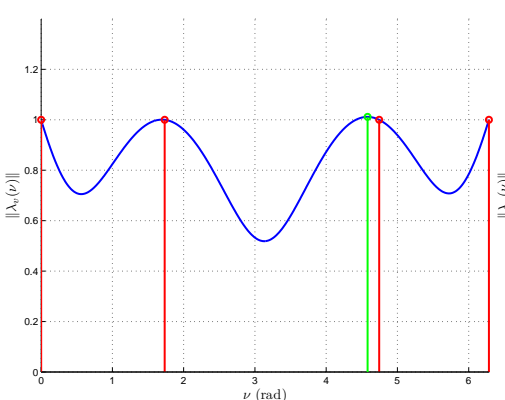

(a) Iteration 4: $v_{b}=v_{m}=4.5814$

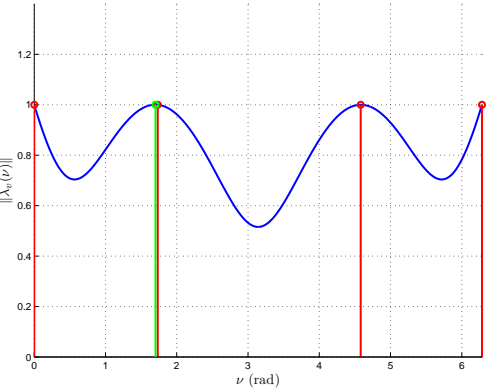

(b) Iteration 5: $v_{b}=v_{m}=1.7017$

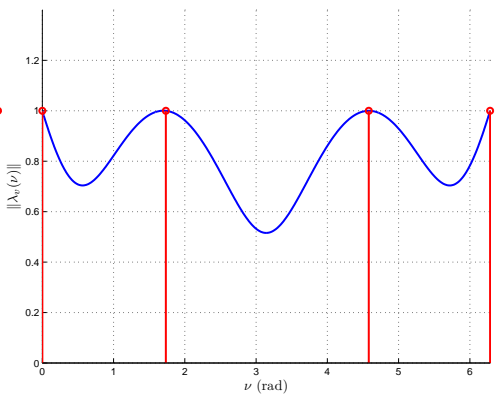

(c) Final norm of primer vector

Fig. 5: Details of iterations 4-6 for the third case of Carter [8]

Relative positions, relative velocities in the LVLH frame and impulses of the optimal plan maneuver are shown on Figures 6a while the Figure $6 \mathrm{~b}$ depicts the trajectory of the chaser in the orbital plane. Only linear simulations based on Hill-Clohessy-

Wiltshire transition matrix have been performed.
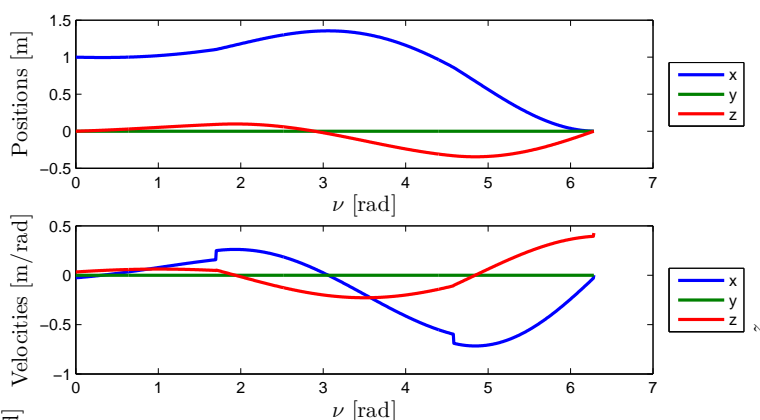

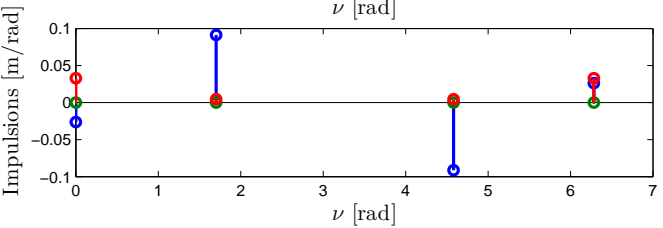

(a) Relatives positions and velocities trajectories and impulses

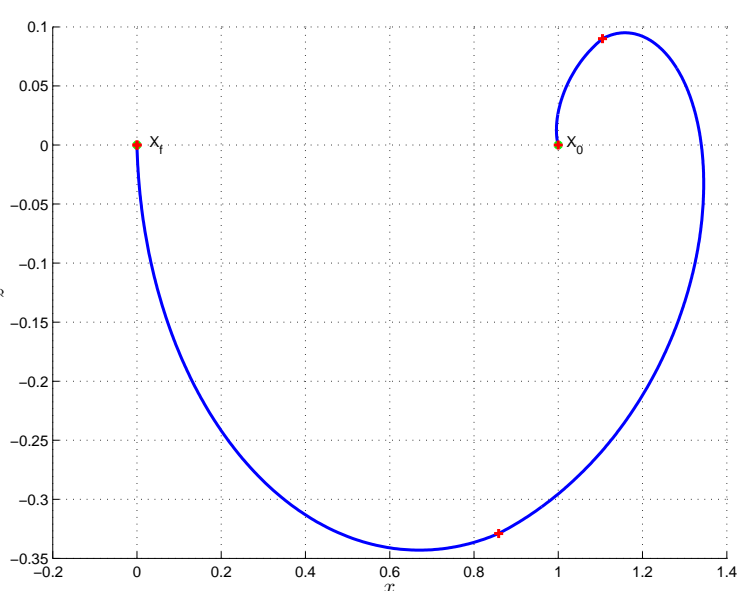

(b) Trajectory of the chaser in the orbital plane $(x, z)$

Fig. 6: State and control trajectories for the Carter's third example

\subsection{Case study 2}

The second numerical illustration is still a coplanar circle-to-circle rendezvous that should be completed within one orbital period and that is borrowed from the reference [8]. The boundary conditions of the rendezvous are modified so that the chaser is one unit above the target with the same initial and final velocities. All characteristics are summarized in Table 3.

\begin{tabular}{|c|c|}
\hline Eccentricity & $e=0$ \\
\hline$v_{0}$ & $0 \mathrm{rad}$ \\
\hline$X_{0}^{T}=\left[\begin{array}{ll}r_{0}^{T} & v_{0}^{T}\end{array}\right]$ & {$\left[\begin{array}{llll}0 & 1 & 0 & 0\end{array}\right]$} \\
\hline$\overline{v_{f}}$ & $2 \pi \mathrm{rad}$ \\
\hline$X_{f}^{T}=\left[\begin{array}{ll}r_{f}^{T} & v_{f}^{\prime}\end{array}\right]$ & {$\left[\begin{array}{llll}0 & 0 & 0 & 0\end{array}\right]$} \\
\hline$N_{\max }$ & 4 \\
\hline
\end{tabular}

Table 3: Carter's second example characteristics 
The resulting optimal trajectory computed with the mixed iterative algorithm is a three-impulse rendezvous as conjectured by Carter. The primer vector trajectory plot depicted in Fig. 7 confirms the optimality of the solution for a fixed number of impulses equal to $N=3$.

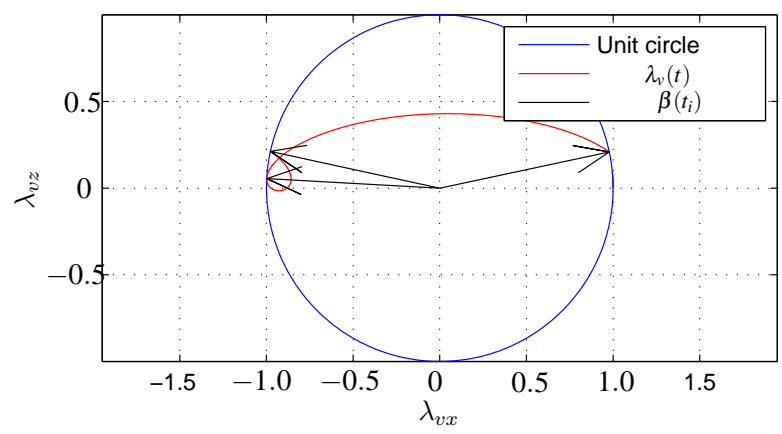

Fig. 7: Primer vector in-plane trajectory for Carter's case study 2 [8]

The optimization process requires 9 iterations during $25 \mathrm{~s}$. These ten steps are detailed in Figures 8 through 10, where each iteration is associated with one particular $T_{\text {imp }}$ update case. As previously, the whole process may be divided into two different stages. The first one is similar to the first example while the second one is made off two alternate operations: Addition of a fourth interior impulse to the previous three-impulse non optimal solution and merging of two interior impulses to get a better three impulse solution. It is important to note that there is no admissible extreme two-impulse solution since the following linear system corresponding to the two-point boundary value problem has no solution.

$$
\left[\begin{array}{cccc}
0 & 2 & 6 \pi & 2 \\
0 & 1 & 0 & 1 \\
2 & 0 & 2 & 0 \\
-1 & 0 & -1 & 0
\end{array}\right]\left[\begin{array}{c}
\Delta V_{x}(0) \\
\Delta V_{z}(0) \\
\Delta V_{x}(2 \pi) \\
\Delta V_{z}(2 \pi)
\end{array}\right]=\left[\begin{array}{c}
0 \\
0 \\
3 \\
-2
\end{array}\right]
$$

and

$$
\left.\operatorname{rank}\left(\left[\begin{array}{cccc}
0 & 2 & 6 \pi & 2 \\
0 & 1 & 0 & 1 \\
2 & 0 & 2 & 0 \\
-1 & 0 & -1 & 0
\end{array}\right]\right)=3<\operatorname{rank}\left(\left[\begin{array}{cccc}
0 & 2 & 6 \pi & 2 \\
0 & 1 & 0 & 1 \\
2 & 0 & 2 & 0 \\
-1 & 0 & -1 & 0
\end{array}\right]\left[\begin{array}{c}
0 \\
0 \\
3 \\
-2
\end{array}\right]\right)\right]=4
$$




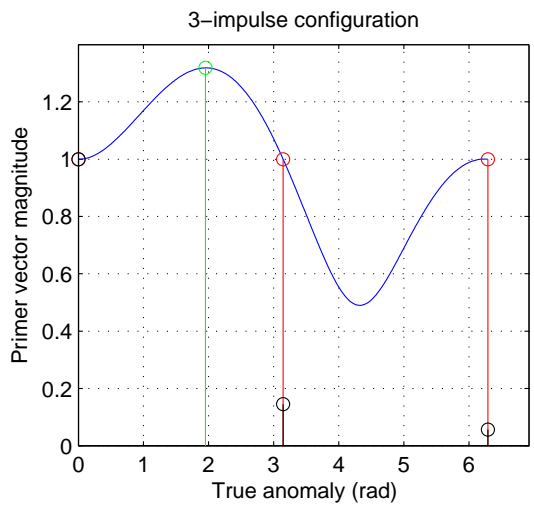

(a) Iter 1: Add impulse at $v=1.950$-Case 8

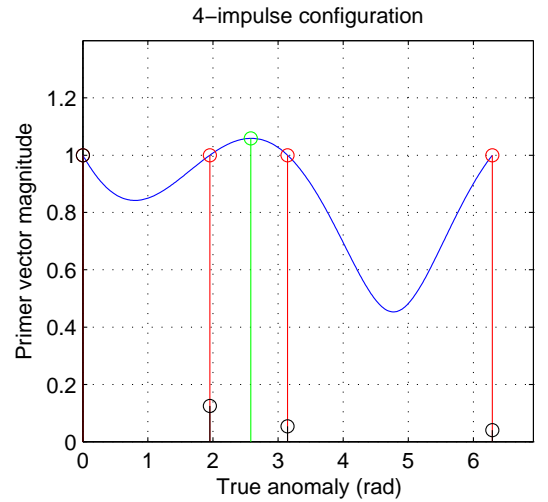

(b) Iter 2: Case 7

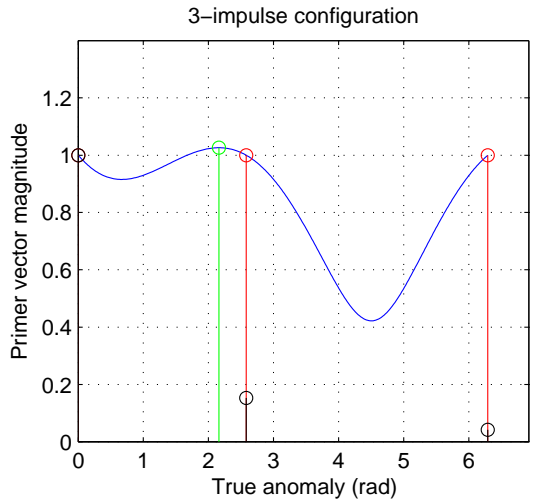

(c) Iter 3: Add impulse at $v=2.559$-Case 8

Fig. 8: Iterations 1-3 details for Carter's case study 2 [8]

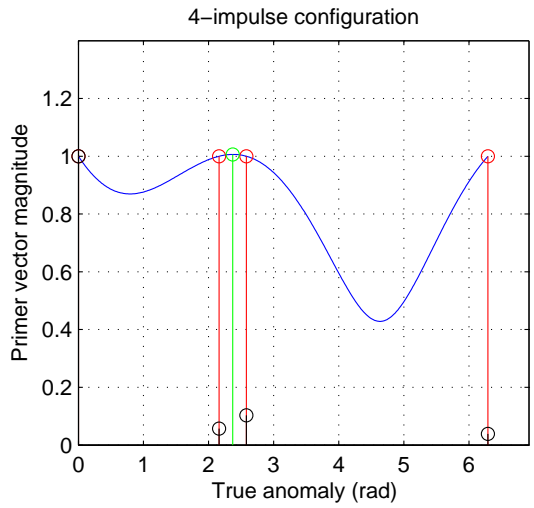

(a) Iter 4: Case 7

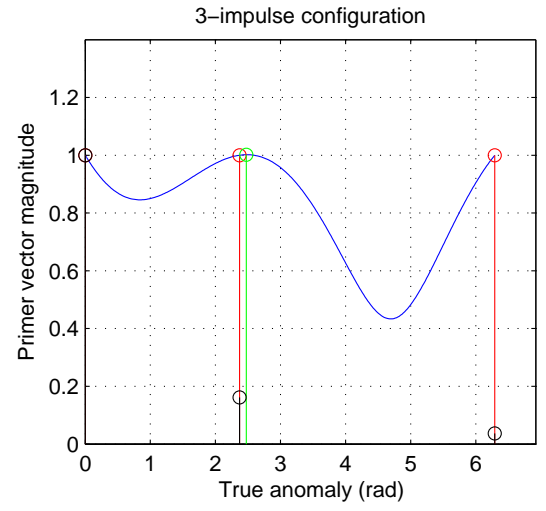

(b) Iter 5: Add impulse at $v=2.475$-Case 8

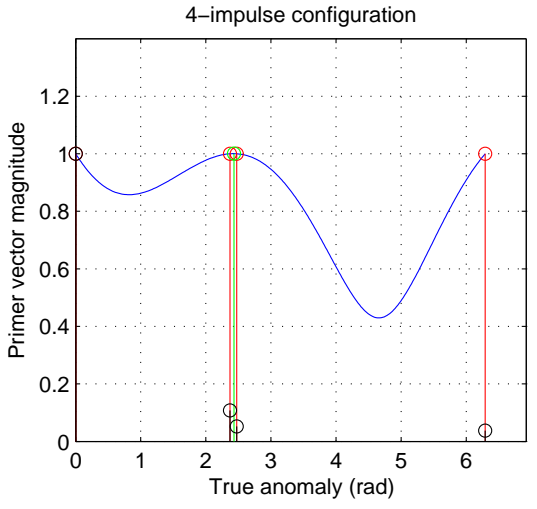

(c) Iter 6: Case 7

Fig. 9: Details of iterations 4-6 for Carter's case study 2 [8]

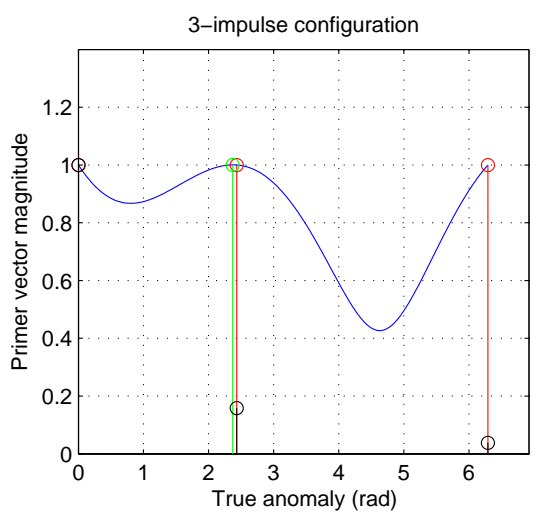

(a) Iter 7: Add impulse at $v=2.370$-Case 8

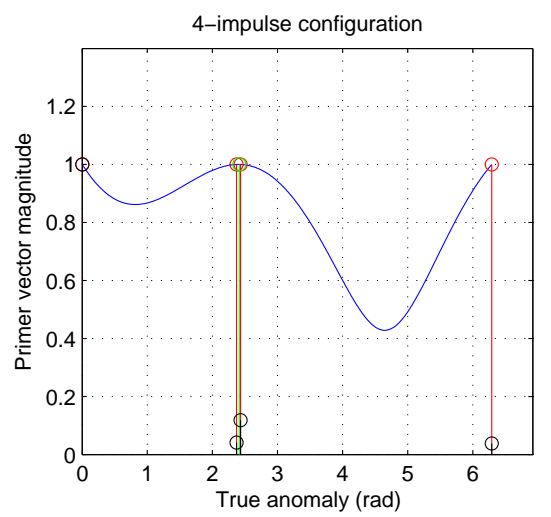

(b) Iter 8: Case 7

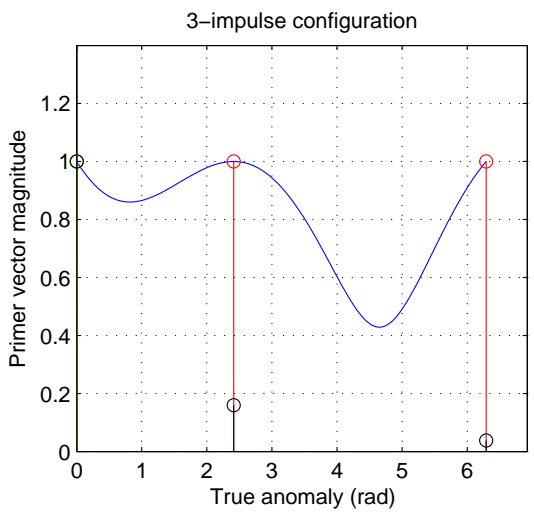

(c) Iter 9: Convergence

Fig. 10: Details of iterations 7-9 for Carter's case study 2 [8]

The results presented in [8] are clearly not optimal with respect to the choice of the date of application of the interior velocity increment. In [8], this location has been chosen a priori and it obviously results in a non optimal consumption as summarized 
in Table 4 where the results of the mixed iterative algorithm are compared to those presented by Carter in [8] with $12 \%$ fuel consumption improvement. Additionally, Table 4 lists the optimality certificate furnished by the PRDV algorithm [20].

\begin{tabular}{|c|c|c|c|}
\hline & Carter [8] & Mixed iterative Algorithm & PRDV algorithm [20] \\
\hline$v_{\text {int }}(\mathrm{rad})$ & $\frac{\pi}{2} \simeq 1.5708$ & 2.4119 & 2.4085 \\
\hline$\Delta V\left(v_{0}\right)^{T}$ & {$[1.6294-0.6667]$} & {$[1.7775-0.3828]$} & {$[1.7771-0.38449]$} \\
\hline$\Delta V\left(v_{1}\right)^{T}$ & {$[0.39010 .0964]$} & {$[0.2896-0.0165]$} & {$[0.28995-0.015971]$} \\
\hline$\Delta V\left(v_{f}\right)^{T}$ & {$[-0.0633-0.0259]$} & {$[-0.0672-0.0143]$} & {$[-0.06706-0.014384]$} \\
\hline $\mathscr{L}_{1} / l_{2}$ cost & 2.2307 & 2.1770 & 2.1772 \\
\hline
\end{tabular}

Table 4: Results from [8], mixed iterative and PRDV algorithms for Carter's case study 2 [20]

Figure 11 shows the in-plane trajectory of the chaser for Carter's solution (green) and mixed iterative algorithm (blue). Interestingly, the simulation of Carter's maneuver planning as proposed in [8] leads to an error at the final point of the rendezvous even with the Hill-Clohessy-Wiltshire state-transition matrix.

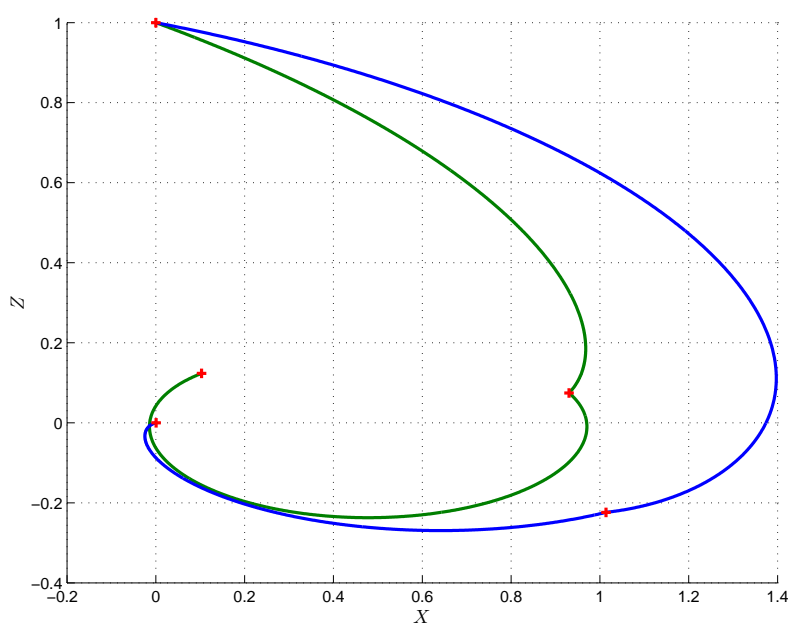

Fig. 11: In-plane trajectory and impulse dates for Carter's case study 2: Mixed iterative algorithm (blue) and Carter's solution (green)

\subsection{Case study 3}

The final academic case of Carter is recalled in the Table 5. As will be seen in the sequel, this simple example is particularly interesting since it exhibits numerical hurdles to find the optimal solution that is quite surprising for so simple an example.

\begin{tabular}{|c|c|}
\hline Eccentricity & $e=0$ \\
\hline$v_{0}$ & $0 \mathrm{rad}$ \\
\hline$X_{0}^{T}$ & {$\left[\begin{array}{llll}1 & 0 & 0 & 0\end{array}\right]$} \\
\hline$v_{f}$ & $2 \pi$ \\
\hline$X_{f}^{T}$ & {$\left[\begin{array}{llll}0 & 0 & 0 & 0\end{array}\right]$} \\
\hline
\end{tabular}

Table 5: Data for Carter's first example [8] 


\subsubsection{Analysis of Carter's solution}

The solution proposed in [8] is a two-impulse solution with an initial and final thrust in opposite directions along the $x$ axis of the LVLH frame. This solution is analytical in the sense that it is the minimum-fuel solution of the linear system:

$$
R_{h c w}(0) \Delta V(0)+R_{h c w}(2 \pi) \Delta V(2 \pi)=\phi_{h c w}^{-1}(2 \pi) X_{f}-\phi_{h c w}^{-1}(0) X_{0}=u_{f}
$$

and may readily be computed as:

$$
\Delta V(0)=\left[\frac{1}{6 \pi} 0\right]^{T} \Delta V(2 \pi)=\left[-\frac{1}{6 \pi} 0\right]^{T}
$$

This conjecture appears to be reasonable if the following result from [18] is recalled.

\section{Theorem 4.1 ([18])}

For HCW rendezvous problems, there is no optimal four-impulse conditions for boundary conditions defined as $X_{0}^{T}=$ $\left[\begin{array}{llll}x_{0} & z_{0} & 0 & 0\end{array}\right], X_{f}^{T}=\left[\begin{array}{llll}0 & 0 & 0 & 0\end{array}\right]$.

Unfortunately, the solution (37) is suboptimal as may be demonstrated by the associated primer vector that does not satisfy all the necessary conditions of optimality. $\lambda_{v}(v)$ is defined by:

$$
\lambda_{v}(v)=R^{T}(v) \lambda
$$

where $\lambda$ is a real solution of the polynomial system (39):

$$
\begin{array}{r}
R(0) R(0)^{T} \Delta v_{0} \lambda+R(2 \pi) R(2 \pi)^{T} \Delta v_{f} \lambda=u_{f} \\
\lambda^{T} R(0) R(0)^{T} \lambda=1 \\
\lambda^{T} R(2 \pi) R(2 \pi)^{T} \lambda=1
\end{array}
$$

minimizing $\lambda^{T} u_{f}$. This minimum-fuel solution $\lambda^{*}$ is given by

$$
\lambda^{*}=\left[\begin{array}{llll}
-0.106103295393226 & 0.212206590785999 & 0.000443462832762 & -0.99912
\end{array}\right]^{T}
$$

The norm and the locus of the primer vector associated to Carter's solution $\lambda_{v}(v)^{c}=R^{T}(v) \lambda^{*}$ are depicted on Figure 12. 


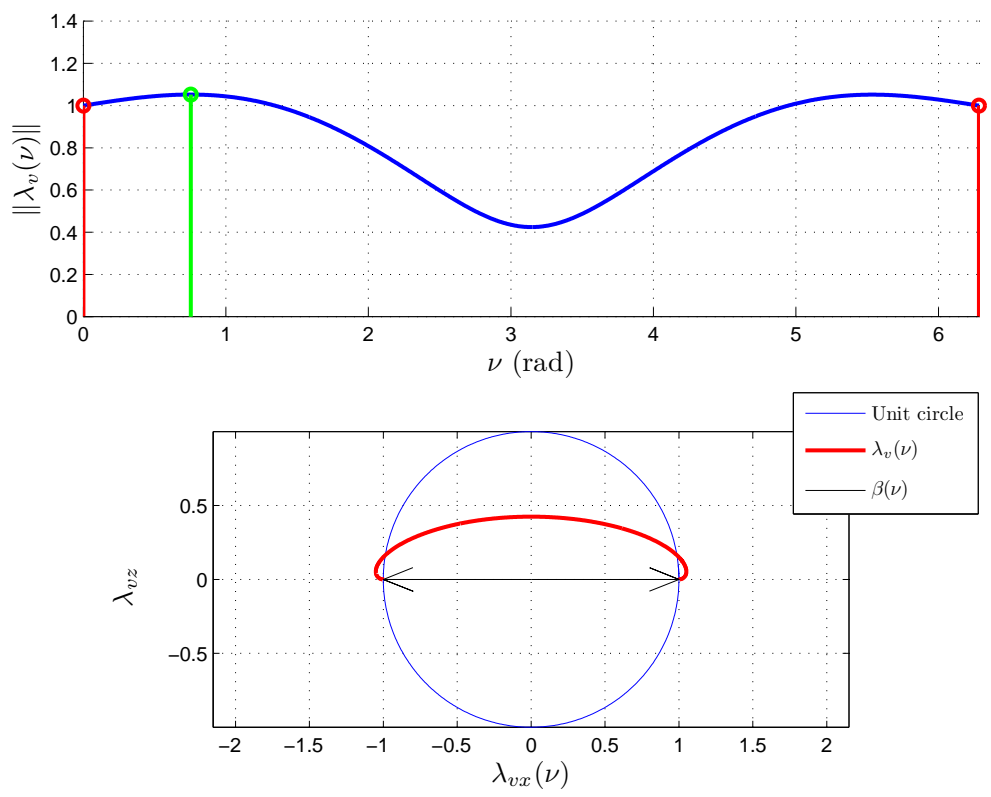

Fig. 12: Norm and primer vector locus for Carter's two-impulse solution

We get that it violates the necessary condition of optimality (9) since its maximum is numerically given by:

$$
\lambda_{v m}^{c}=\max _{v \in\left[v_{1}, v_{f}\right]}\left\|\lambda_{v}(v)^{c}\right\|=1.052327525631438
$$

The sub-optimality of Carter's solution may be formally proved by considering that $\lambda^{*}$ must be the solution of the following linear system:

$$
\left[\begin{array}{cccc}
36 \pi^{2}+8 & 4 & 12 \pi & -6 \pi \\
4 & 2 & 0 & 0 \\
12 \pi & 0 & 8 & -4 \\
-6 \pi & 0 & -4 & 2
\end{array}\right]\left[\begin{array}{c}
\lambda_{1} \\
\lambda_{2} \\
\lambda_{3} \\
\lambda_{4}
\end{array}\right]=\left[\begin{array}{c}
-6 \pi \\
0 \\
0 \\
0
\end{array}\right]
$$

Hence, $\lambda^{*}$ may be parametrized as:

$$
\lambda^{*}=\left[-\frac{1}{3 \pi} \frac{2}{3 \pi} \lambda_{3}-1+2 \lambda_{3}\right]^{T}
$$

If $\lambda^{*}$ is a solution verifying the optimality condition (9) then

$$
\left\|\lambda_{v}(v)^{c}\right\|=\left\|R_{h c w}^{T}(v) \lambda^{*}\right\|<1, \quad \forall v \in(0,2 \pi)
$$


The condition (44) is equivalent to the existence of $\lambda_{3} \in \mathbb{R}$ such that $\forall v \in(0,2 \pi)$ :

$$
\begin{aligned}
\left\|\lambda_{v}(v)\right\|^{2}= & \left\|R_{h c w}^{T}(v) \lambda\right\|^{2}<1 \\
= & {\left[4(\cos v-1)^{2}+\sin ^{2} v\right] \lambda_{3}^{2}-\frac{4}{\pi}(\cos v-1)[v-\pi-\sin v] \lambda_{3} } \\
& +\frac{(3(v-\pi)-4 \sin v)^{2}+4(\cos v-1)^{2}}{9 \pi^{2}}<1
\end{aligned}
$$

For $v=\frac{\pi}{20}$, it is easy to show that the second order polynomial of (45) is always strictly greater than $1, \forall \lambda_{3} \in \mathbb{R}$. Carter's solution is therefore not optimal.

\subsubsection{Analytical two-impulse solution}

Let us now consider this problem but without setting the two times of thrusting a priori but by defining $\left(v_{1}, v_{2}\right)$ as decision variables. The two-point boundary value problem reads as:

$$
X_{f}-\Phi(2 \pi, 0) X_{0}=\Phi\left(2 \pi, v_{1}\right) B \Delta V\left(v_{1}\right)+\Phi\left(2 \pi, v_{2}\right) B \Delta V\left(v_{2}\right)
$$

and is equivalent to the system (47) to be solved:

$$
\left.\begin{array}{rrrr}
-6 \pi+3 v_{1}-4 \sin v_{1} & 2-2 \cos v_{1} & -6 \pi+3 v_{2}-4 \sin v_{2} & 2-2 \cos v_{2} \\
-2+2 \cos v_{1} & -\sin v_{1} & -2+2 \cos v_{2} & -\sin v_{2} \\
-3+4 \cos v_{1} & -2 \sin v_{1} & -3+4 \cos v_{2} & -2 \sin v_{2} \\
2 \sin v_{1} & \cos v_{1} & 2 \sin v_{2} & \cos v_{2}
\end{array}\right]\left[\begin{array}{c}
\Delta V_{x}\left(v_{1}\right) \\
\Delta V_{z}\left(v_{1}\right) \\
\Delta V_{x}\left(v_{2}\right) \\
\Delta V_{z}\left(v_{2}\right)
\end{array}\right]=\left[\begin{array}{c}
-1 \\
0 \\
0 \\
0
\end{array}\right.
$$

The determinant of $M\left(v_{1}, v_{2}\right)$ is:

$$
\operatorname{det} M\left(v_{1}, v_{2}\right)=16 \sin ^{2}\left(\left(v_{2}-v_{1}\right) / 2\right)-3\left(v_{2}-v_{1}\right) \sin \left(v_{2}-v_{1}\right)=g(\theta)
$$

where $0 \leq \theta=v_{2}-v_{1} \leq 2 \pi$. The function $g(\theta)$ is a strictly positive on $(0,2 \pi)$ and vanishes for 0 and $2 \pi$ as may be easily verified [6]. Cases $\theta=0$ and $\theta=2 \pi$ may be excluded for obvious reasons. We get the vector of thrusts as functions of $\left(v_{1}, v_{2}\right)$.

$$
\left[\begin{array}{c}
\Delta V_{x}\left(v_{1}\right) \\
\Delta V_{z}\left(v_{1}\right) \\
\Delta V_{x}\left(v_{2}\right) \\
\Delta V_{z}\left(v_{2}\right)
\end{array}\right]=M^{-1}(\theta) \varpi_{f}=\frac{1}{g(\theta)}\left[\begin{array}{c}
-\sin \theta \\
-4 \sin ^{2}(\theta / 2) \\
\sin \theta \\
-4 \sin ^{2}(\theta / 2)
\end{array}\right]
$$


The $\mathscr{L}_{1} / l_{2}$ minimum-fuel rendezvous problem is nothing but the parametric optimization problem (50) where $\theta$ is the only decision variable.

$$
\begin{aligned}
& \min _{\theta} \frac{2 \sqrt{\sin ^{2} \theta+16 \sin ^{4}(\theta / 2)}}{16 \sin ^{2}(\theta / 2)-3 \theta \sin \theta}=J_{l_{2}}(\theta) \\
& \text { s.t. } 0<\theta<2 \pi
\end{aligned}
$$

As it is, this problem appears difficult to solve analytically. In fact, (50) is a convex optimization problem for which a minimum may be computed via the computation of the only zero of its derivative (51) on the interval $(0,2 \pi)$ by a Newton method.

$$
\frac{d J_{l_{2}}(\theta)}{d \theta}=-2 \frac{18 \theta+8 \sin (2 \theta)-9 \sin (3 \theta) / 4-37 \sin \theta / 4+24 \theta\left(2 \sin ^{2}(\theta / 2)-1\right)-6 \theta\left(2 \sin ^{2}(\theta)-1\right)}{\left(16 \sin ^{2}(\theta / 2)-3 \theta \sin \theta\right)^{2} \sqrt{16 \sin ^{4}(\theta / 2)+\sin ^{2} \theta}}
$$

We get the minimizer $\theta^{*}$ of (50) as

$$
\theta^{*}=6.230033575529312
$$

The first and second derivatives of $g(\theta)$ are shown on Figures 13a and 13b confirming the convexity of the problem on the interval $(0,2 \pi)$.

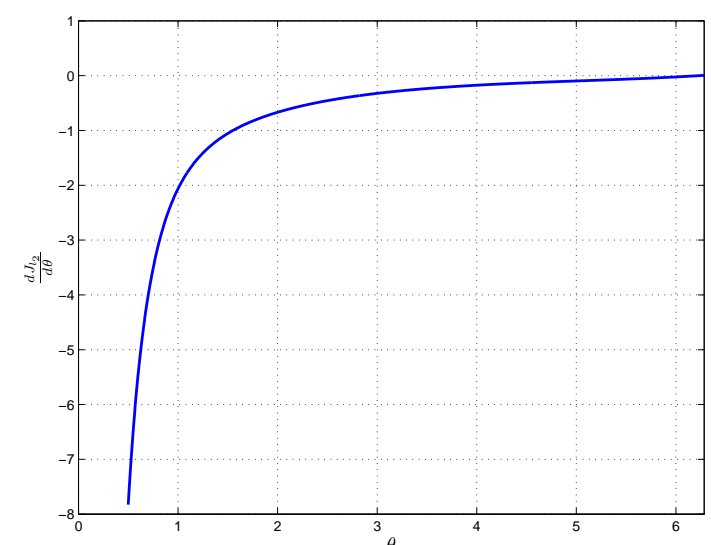

(a) $\frac{d J_{l_{2}}(\theta)}{d \theta}$

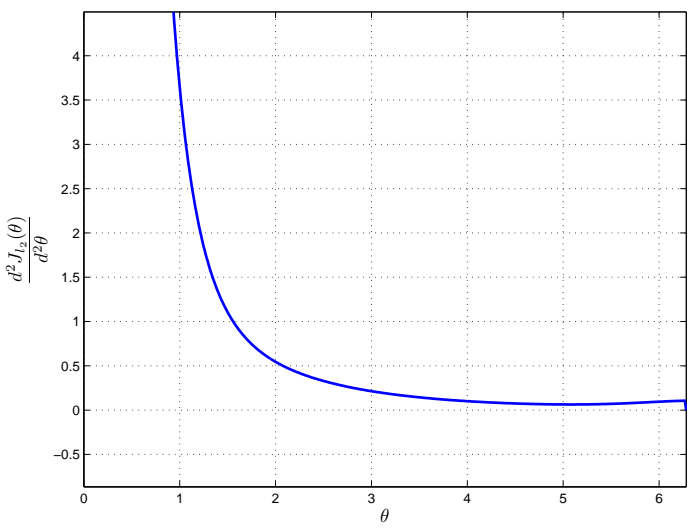

(b) $\frac{d^{2} J_{2}(\theta)}{d \theta^{2}}$

Fig. 13: $\frac{d J_{l_{2}}(\theta)}{d \theta}$ and $\frac{d^{2} J_{l_{2}}(\theta)}{d \theta^{2}}$ on $(0,2 \pi)$

Finally, the optimal cost and the optimal maneuvers for Carter's example are given by:

$$
J_{l_{2}}\left(\theta^{*}\right)=0.105954087364712\left[\begin{array}{l}
\Delta V_{x}\left(v_{1}\right) \\
\Delta V_{z}\left(v_{1}\right) \\
\Delta V_{x}\left(v_{2}\right) \\
\Delta V_{z}\left(v_{2}\right)
\end{array}\right]=\left[\begin{array}{c}
-0.052902333870518 \\
-0.002812512822111 \\
0.052902333870518 \\
-0.002812512822111
\end{array}\right]
$$


These results show that the optimal two-impulse solution for this example is non unique. There is an infinity of optimal solutions with the same consumption and a couple of optimal impulse times verifying $\left(v_{1}^{*}, v_{2}^{*}\right) \in\left(0,2 \pi-\theta^{*}\right) \times\left(\theta^{*}, 2 \pi\right)$ with $v_{2}^{*}-v_{1}^{*}=\theta^{*}$.

\subsubsection{The mixed iterative algorithm solution}

The results of the mixed iterative algorithm are presented in the Table 6 and clearly show that Carter's solution is indeed not an optimal solution. The mixed algorithm converges after 13 iterations to a four-impulse solution illustrated by the Figure 15 a.

\begin{tabular}{|c|c|}
\hline & Mixed iterative Algorithm \\
\hline$v_{i}(\mathrm{rad})$ & $00.06816 .18372 \pi]$ \\
\hline$\Delta V\left(v_{0}\right)^{T}$ & {$[0.0214530 .001138]$} \\
\hline$\Delta V\left(v_{1}\right)^{T}$ & {$[0.0314490 .001685]$} \\
\hline$\Delta V\left(v_{2}\right)^{T}$ & {$[0.0067970 .000364]$} \\
\hline$\Delta V\left(v_{f}\right)^{T}$ & {$[0.0461050 .002446]$} \\
\hline $\mathscr{L}_{1} / l_{2}$ Cost & 0.105954 \\
\hline
\end{tabular}

Table 6: Results of the mixed iterative algorithm for Carter's first example [8]

The norm of the primer vector is given on Figure 14 where $\lambda_{v m}(v)=1.0000048$.
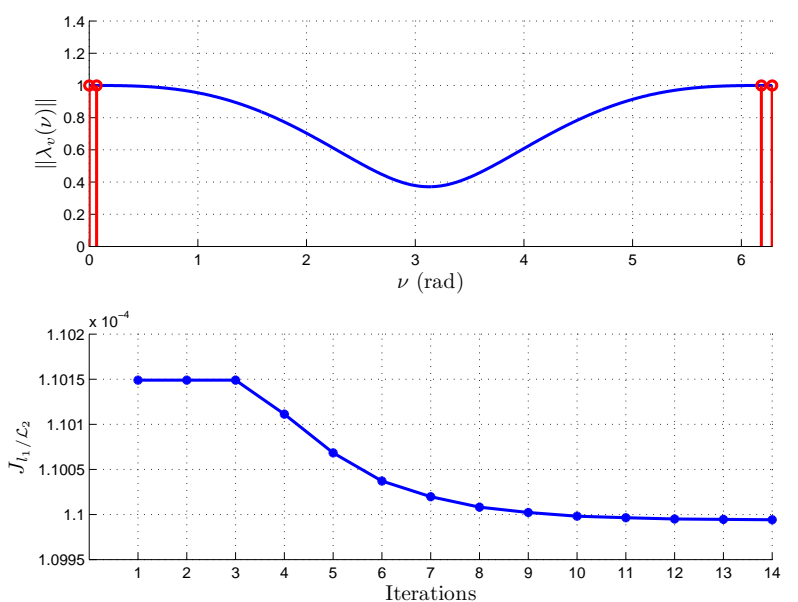

Fig. 14: Norm of the primer vector and cost evolution

The distribution of this solution may be defined as two pairs of almost simultaneous impulses at the beginning and at the end of the rendezvous (as is illustrated on the plot of the trajectory in the orbital plane at Figure 15b). 

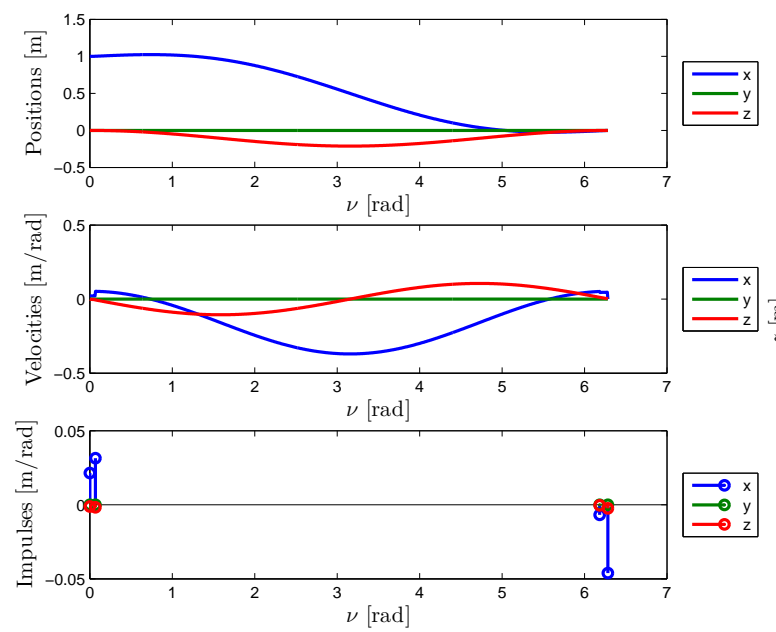

(a) Relatives positions and velocities trajectories and impulses

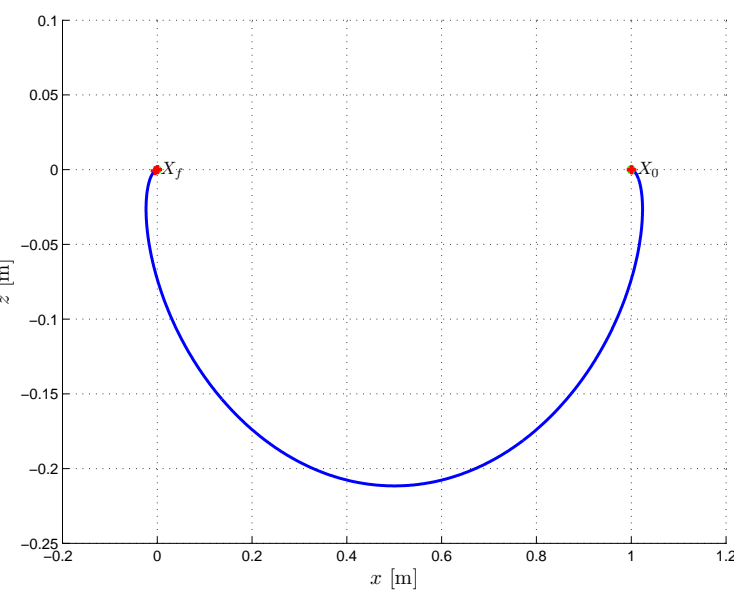

(b) Trajectory of the chaser in the orbital plane $(x, z)$

Fig. 15: State and control trajectories for the Carter's first case [8]

Keeping in mind that the optimal solution has been computed analytically as a two-impulse solution with one initial and one final coasting period and recalling the result presented by theorem 4.1 , the result of the mixed iterative algorithm may be analyzed as a tight approximation of the genuine optimal two-impulse solution.

\subsection{Case study 4}

Following the first three academic numerical examples, a more realistic illustration based on PRISMA [3] is now presented. PRISMA programme is a cooperative effort between the Swedish National Space Board (SNSB), the French Centre National d'Etudes Spatiales (CNES), the German Deutsche Zentrum für Luft- und Raumfahrt (DLR) and the Danish Danmarks Tekniske Universitet (DTU) [2]. Launched on June 15, 2010 Yasny (Russia), it was intended to test in-orbit new guidance schemes (particularly autonomous orbit control) for formation flying and rendezvous technologies. This mission includes the FFIORD experiment led by CNES which features a rendezvous maneuver (formation acquisition). The orbital elements of the target orbit as well as initial and final rendezvous conditions are listed in Table 7.

\begin{tabular}{|c|c|}
\hline Semi-major axis & $a=7011 \mathrm{~km}$ \\
\hline Inclination & $i=98$ deg. \\
\hline Argument of Perigee & $\omega=0 \mathrm{deg}$. \\
\hline Right Ascension of the Ascending Node & $\Omega=190 \mathrm{deg}$. \\
\hline Eccentricity & $e=0.004$ \\
\hline True Anomaly & $v_{0}=0 \mathrm{rad}$. \\
\hline$t_{0}$ & $0 \mathrm{~s}$ \\
\hline$X_{0}^{T}=\left[\begin{array}{ll}r_{0}^{T} & v_{0}^{T}\end{array}\right]$ & {$\left[\begin{array}{lllll}-1 & 0 & 0 & 0 & 0\end{array}\right] \mathrm{km}-\mathrm{km} / \mathrm{s}$} \\
\hline$t_{f}$ & $64620 \mathrm{~s}$ \\
\hline$X_{f}^{T}=\left[\begin{array}{ll}r_{f}^{T} & v_{f}^{T}\end{array}\right]$ & {$\left[\begin{array}{llll}-100 & 0 & 0 & 0\end{array}\right] \mathrm{m}-\mathrm{m} / \mathrm{s}$} \\
\hline$N_{\max }$ & 4 \\
\hline
\end{tabular}

Table 7: PRISMA rendezvous characteristics 
To save fuel and allow for in-flight testing throughout the FFIORD experiment, the rendezvous maneuver must last several orbits. Duration of the rendezvous is approximately 12 hours for an expected average cost of $20 \mathrm{~cm} / \mathrm{s} \mathrm{[3].}$

The mixed iterative algorithm achieves optimization within 13 seconds and within 3 iterations. Global optimality of this three-impulse solution has been established in [20] by running the PDRV algorithm.

\begin{tabular}{|c|c|c|}
\hline & PRDV Algorithm & Mixed iterative algorithm \\
\hline$t_{\text {int }}(\mathrm{s})$ & 3189.3 & 3198.6 \\
\hline$v_{\text {int }}(\mathrm{rad})$ & 3.4285 & 3.4377 \\
\hline$\Delta V\left(v_{0}\right)^{T}$ & {$[-0.049110 .002152]$} & {$[-0.049110 .001933]$} \\
\hline$\Delta V\left(v_{1}\right)^{T}$ & {$[-0.0020380 .0000099]$} & {$[-0.0020390 .0000112]$} \\
\hline$\Delta V\left(v_{f}\right)^{T}$ & {$[0.0513150 .001423]$} & {$[0.0513160 .001404]$} \\
\hline $\mathscr{L}_{1} / l_{2}$ Cost $\mathrm{m} / \mathrm{s}$ & 0.102525 & 0.10252 \\
\hline
\end{tabular}

Table 8: Results of the mixed iterative algorithm and PRDV algorithm [20] for the PRISMA case study

Figure 16b shows primer vector magnitude during transfer. Note the low magnitude of the second impulse $(0.002 \mathrm{~m} / \mathrm{s})$ with respect to the initial and final velocity increments $(0.0492 \mathrm{~m} / \mathrm{s}$ and $0.0513 \mathrm{~m} / \mathrm{s})$ but these velocity increments play a significant role in the optimality of the result. In particular, they provide the right chaser orientation for the long drift (61400 s) between the second impulse and the final one. Indeed, the designer could be tempted to remove this interior impulse and resort to the suboptimal two-impulse strategy. The latter solution proves to be strongly suboptimal since its $\mathscr{L}_{1} / l_{2}$ cost is $27 \%$ greater than the optimal solution $(0.14506 \mathrm{~m} / \mathrm{s})$. The long drifting period of $61400 \mathrm{~s}$ of the optimal solution is clearly illustrated in Figure 16a where the in-plane trajectory and impulse positions are represented. Finally, it is worth noticing that the optimal cost is half the expected average cost of $20 \mathrm{~cm} / \mathrm{s}$ [3].

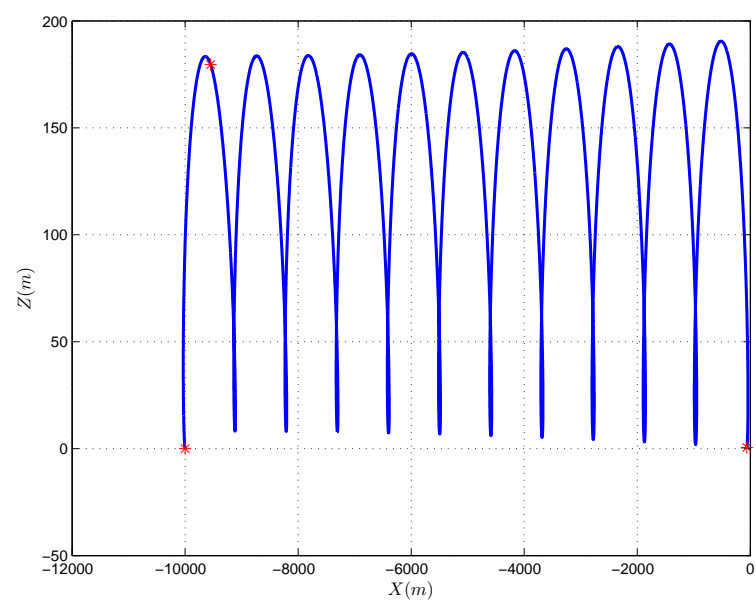

(a) In-plane trajectory and impulse positions * for PRISMA mission

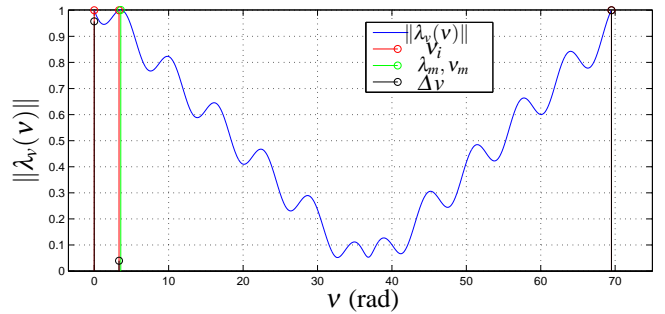

(b) Primer vector magnitude during PRISMA mission

Fig. 16: Primer vector norm and trajectory for the PRISMA mission

\section{Conclusion}

A new numerical algorithm based on heuristic rules deduced from the work of [10] and tools from algebraic geometry has been proposed to address the issue of time-fixed optimal rendezvous in a linear setting. This algorithm is a mixed iterative 
algorithm optimizing over the number of impulses with a low numerical complexity mainly consisting in the solution of a small size polynomial system of equations. Even without a formal proof of convergence, this heuristic algorithm appears to be very efficient in practice on very different missions ranging from circular to elliptic rendezvous missions. In particular, it is not necessary to resort to some local optimization scheme as in the references [11] and [12] to eliminate cusp occurrences in the graph of the norm of the primer vector.

Despite the good numerical results presented, some improvement can still be expected if more sophisticated transition matrices including orbital perturbation effects are used such as atmospheric drag or gravity harmonics term $\mathbf{J}_{2}$.Another avenue of research deals with the extension of this algorithm for optimal trajectory planning with collision avoidance constraints.

\section{References}

1. Hughes, S. P., Mailhe, L. M., and Guzman, J. J.: A comparison of trajectory optimization methods for the impulsive minimum fuel rendezvous problem. In: Gravseth, I. J., Culp, R. D. (eds): Advances in Guidance and Control, vol. 113, pp. 85-104. Advances in the Astronautical Sciences, AIAA, New York, (2003)

2. Larsson, R., Berge, S., Bodin, P., and Jonsson, U.: Fuel Efficient Relative Orbit Control Strategies for Formation Flying and Rendezvous within PRISMA. In: Proceedings of the 29th Annual AAS Rocky Mountain Guidance and Control Conference, Breckenridge, Colorado, USA, pp. 25-40 (2006)

3. Berges, J. C., Cayeux, P., Gaudel-Vacaresse, A., and Messygnac, B.: CNES approaching guidance experiment within FFIORD. In: Proceedings of the $21^{\text {st }}$ International Symposium on Space Flight Dynamics, Annapolis, Maryland, USA, 24-28 (2007)

4. Delpech, M. Berges, J. C., Djalal, S., Guidotti, P. Y., Christy, J.: Preliminary results of the vision based rendezvous and formation flying experiments performed during the PRISMA extended mission. In: Proceedings of the $1^{\text {st }}$ IAA Conference on Dynamics and Control of Space Systems, DYCoSS’ 2012, Porto, Portugal (2012)

5. Lawden, D. F.: Optimal trajectories for space navigation. Butterworth, London (1963)

6. Prussing, J. E.: Optimal multiple-impulse orbital rendezvous. Ph.D. Thesis, Massachusetts Institute of Technology, Cambridge, MA (1967)

7. Prussing, J. E.: Illustration of the primer vector in time-fixed orbit transfer. AIAA Journal 7(6), 1167-1168 (1969)

8. Carter, T. E.: Optimal impulsive space trajectories based on linear equations. J. Optim. Theory and Appl. 70(2), 277-297 (1991)

9. Carter, T. E.: Necessary and sufficient conditions for optimal impulsive rendezvous with linear equations of motion. Dynamics and control. 10, $219-227(2000)$

10. Lion, P. M., and Handelsman, M.: Primer vector on fixed-time impulsive trajectories. AIAA Journal. 6(1). 127-132 (1968)

11. Jezewski, D. J., and Rozendaal, H.L.: An efficient method for calculationg optimal free-space n-impulse trajectories. AIAA Journal. 6(11), 2160$2165(1968)$

12. Jezewski, D. J.: Primer vector theory applied to the linear relative-motion equations. Optimal control applications and methods. 1, 387-401 (1980)

13. Prussing, J. E., Chiu, J. H.: Optimal multiple-impulse time-fixed rendezvous between circular orbits. Journal of Guidance, Control and Dynamics 9(1), 17-22 (1986)

14. Tschauner, J., and Hempel, P., "Rendevous zu einem in elliptischer Bahn umlaufenden Ziel," Astronautica Acta, Vol. II, No. 2, 1965, pp. 104-109.

15. Yamanaka, K., and Ankersen, F.: New state transition matrix for relative motion on an arbitrary elliptical orbit. Journal of Guidance, Control and Dynamics. 25(1), 60-66 (2002)

16. Kara-Zaitri, M. Arzelier, D., and Louembet, C.: Mixed iterative algorithm for solving impulsive time-fixed rendezvous problem. In: Proceedings of the AIAA Guidance, Navigation, and Control Conference, Toronto, Canada (2010) 
17. Carter, T. E., and Brient, J.: Linearized impulsive rendezvous problem. J. of Optim. Theory and Appl. 86(3), 553-584 (1995)

18. Carter, T. E., Alvarez, S. A.: Quadratic-based computation of four-impulse optimal rendezvous near circular orbit: Journal of Guidance, Control and Dynamics. 23(1), 109-117 (2000)

19. Prussing, J. E.: Optimal impulsive linear systems: Sufficient conditions and maximum number of impulses. Journal of the Astronautical Sciences. 43(2), 195-206 (1995)

20. Arzelier, D., Kara-Zaitri, M., Louembet, C., Delibasi, A.: Using polynomial optimization to solve the fuel-optimal impulsive rendezvous problem. Journal of Guidance, Control and Dynamics. 34(5), 1567-1572 (2011)

21. Neustadt, L. W.: A general theory of minimum-fuel space trajectories. SIAM Journal of Control. 3(2), 317-356 (1965)

22. Guzmán, J. J., Mailhe, L. M., Schiff, C., Hughes, S. P., Folta, D. C.: Primer vector optimization: Survey of theory, new analysis and applications. In: Proceedings of the 53 ${ }^{\text {rd }}$ International Astronautical Congress, Houston, Texas, USA, 10-19 (2002)

23. Verschelde, J.: Homotopy methods for solving polynomial systems. In: Proceedings of the International Symposium on Symbolic and Algebraic Computations, Beijing, China (2005)

24. Verschelde, J.: Algorithm 795: PHCpack: A general-purpose solver for polynomial systems by homotopy continuation. ACM Transactions on Mathematic Software. 25(2), 251-276 (1999)

25. Clohessy, W. H., and Wiltshire, R. S.: Terminal guidance system for satellite rendezvous. Journal of the Aerospace Sciences. 27(9), 653-658 (1960) 